\title{
THE GEOMETRY OF PATHS*
}

\author{
BY \\ OSWALD VEBLEN AND TRACY YERKES 'IHOMAS
}

1. Introduction. The first part of this paper is intended as a systematic general account of the geometry of paths and is largely based on the series of notes by Eisenhart and Veblen in volume 8 of the Proceedings of the National Academy of Sciences. The general theory is carried far enough to include an account of a series of tensors defined by means of normal coördinates, and also a series of generalizations of the operation of covariant differentiation. We then turn to a special problem, the investigation of the conditions which must be satisfied by the functions $\Gamma$ in order that the differential equations of the paths shall possess homogeneous first integrals. $t$ We first solve a still more special problem for first integrals of the $n$th degree $(\S 15)$. This includes as a special case the problem solved by Eisenhart and Veblen in the Proceedings of the National Academy of Sciences, vol. 8 (1922), p. 19, of finding the conditions which must be satisfied by the $\Gamma$ 's in order that the equations (2.1) shall be the differential equations of the geodesics of a Riemann space.

Finally we solve the general problem for the linear and quadratic cases; that is to say, we find algebraic necessary and sufficient conditions on the functions $\boldsymbol{\Gamma}$ in order that (2.1) shall possess homogeneous linear and quadratic first integrals. The method used will generalize to homogeneous first integrals of the $n$th degree. We leave unsolved all the projective problems which correspond to the affine problems which we have solved. For example, the problem remains open to find what condition must be satisfied by the $\Gamma$ 's in order that one of the sets of differential equations which define the same paths as (2.1) shall have a linear first integral.

2. The geometry of paths. Consider an $n$-dimensional region the points of which can be represented by coördinates $\left(x^{1}, x^{2}, \ldots, x^{n}\right)$. Also consider a set of differential equations

* Presented to the Society, October 28, 1922, and April 28, 1923.

† Our problem is distinguished from the problem of the existence of first integrals in dynamical systems (studied by Staeckel, Painlevé, Levi-Civita, and others) by the fact that the dynamical problem presupposes the existence of the integral corresponding to the fundamental quadratic form. Cf. Ricci and Levi-Civita, Méthodes de calcul différentiel absolu, Mathematische Annalen, vol. 54 (1901), p. 125. 


$$
\frac{d^{2} x^{i}}{d s^{2}}+\Gamma_{\kappa \beta}^{i} \frac{d x^{\alpha}}{d s} \frac{d x^{\beta}}{d s}=0
$$

in which

$$
\Gamma_{j k}^{i}=\boldsymbol{I}_{k j}^{i}
$$

In these expressions the subscripts and superscripts take all integer values from 1 to $n$ and the convention is employed that any term which contains the same index twice, once as a subscript and once as a superscript, represents a summation with respect to every such index. Thus the second term represents a quadratic form in $d x^{i} / d s$. The coefficients are arbitrary analytic functions of $\left(x^{1}, x^{2}, \ldots, x^{n}\right)$. The condition (2.2) is no restriction on the differential equations (2.1) because the coefficients of any quadratic form can be written so as to satisfy (2.2).

Any curve

$$
x^{i}=\psi^{i}(s)
$$

which satisfies (2.1) is called a path and the theory of these paths is what we call the geometry of paths.

The geometry of paths is a natural generalization of the euclidean geometry. For the differential equations of the straight lines in an $n$-dimensional euclidean space are

$$
\frac{d^{2} x^{i}}{d s^{\mathbf{y}}}=0
$$

when referred to a cartesian coördinate system. An arbitrary transformation of the coördinates

$$
x^{i}=y^{i}\left(y^{1}, y^{2}, \ldots, y^{n}\right)
$$

transforms (2.4) into a set of differential equations of the form (2.1) in the variables $y$, in which

$$
\Gamma_{j k}^{i}=\frac{\partial y^{i}}{\partial x^{\alpha}} \frac{\partial^{2} x^{\alpha}}{\partial y^{j} \partial y^{k}} .
$$

Hence the system of paths defined by (2.1) has the properties of the straight lines of euclidean space whenever the functions $\Gamma$ are such that (2.1) can be transformed by an analytic transformation into (2.4). This transformation is possible if and only if

$$
\frac{\partial \Gamma_{j k}^{i}}{\partial x^{l}}-\frac{\partial \Gamma_{j l}^{i}}{\partial x^{k}}+\Gamma_{j k}^{\alpha} \Gamma_{\alpha l}^{i}-\Gamma_{j l}^{\alpha} \Gamma_{\alpha k}^{i}=0,
$$


as can easily be proved. The left member of this equation is denoted by $B_{j k l}^{i}$ and is called the curvature tensor.

The paths defined by (2.1) are the geodesics of a Riemann space in case the $\boldsymbol{r}$ 's are such that there exists a quadratic differential form

such that

$$
d s^{2}=g_{\alpha \beta} d x^{\alpha} d x^{\beta}
$$

$$
\frac{\partial g_{i j}}{\partial x^{k}}-g_{i \alpha} \Gamma_{j k}^{\alpha}-g_{j \alpha} \Gamma_{i k}^{\alpha}=0
$$

In this case the paths are the geodesics of the differential form (2.8).

The geometry of paths reduces to a Weyl metric geometry if the $\Gamma$ 's are such that there exists a linear form $\varsigma_{\alpha} d x^{\alpha}$ and a quadratic form $g_{\alpha \beta} d x^{\alpha} d x^{\beta}$ such that

$$
g_{\alpha k} \Gamma_{i j}^{\alpha}=\frac{1}{2}\left(\frac{\partial g_{i k}}{\partial x^{j}}+\frac{\partial g_{j k}}{\partial x^{i}}-\frac{\partial g_{i j}}{\partial x^{k}}\right)+\frac{1}{2}\left(g_{i k} \varphi_{j}+g_{j k} \varphi_{i}-g_{i j} \varphi_{k}\right)
$$

(cf. H. Weyl, Raum, Zeit, Materie, 4th edition, p. 113).

In the general case (no restriction on the $\Gamma$ 's except (2.2)) the geometry of paths is equivalent to the geometry of infinitesimal parallelism as developed by Weyl, in Raum, Zeit, Materie (4th edition, p. 100). For any system of $\Gamma$ 's which appear in the differential equations (2.1) can be used to establish a definition of infinitesimal parallelism according to which the paths defined by (2.1) are geodesics in the sense of Weyl.

3. Transformation of the dependent variables. Consider an arbitrary analytic transformation of the coordinates

$$
\bar{x}^{i}=f^{i}\left(x^{1}, x^{2}, \ldots, x^{n}\right)
$$

which may also be written

$$
x^{i}=g^{i}\left(\bar{x}^{1}, \bar{x}^{\mathbf{2}}, \ldots, \bar{x}^{n}\right) .
$$

By substituting (2.3) in (3.1) we obtain

$$
\bar{x}^{i}=\Psi^{i}(s)
$$

as the equation of the path represented by (2.3). Since

$$
\frac{d x^{i}}{d s}=\frac{\partial x^{i}}{\partial \bar{x}^{\alpha}} \frac{d \bar{x}^{\alpha}}{d s}
$$


and

$$
\frac{d^{2} x^{i}}{d s^{2}}=\frac{\partial^{2} x^{i}}{\partial \bar{x}^{\alpha} \partial \bar{x}^{\beta}} \frac{d \bar{x}^{\alpha}}{d s} \frac{d \bar{x}^{\beta}}{d s}+\frac{\partial x^{i}}{\partial \bar{x}^{\alpha}} \frac{d^{2} \bar{x}^{\alpha}}{d s^{2}}
$$

we find that this path satisfies the differential equation

$$
\frac{d^{2} \bar{x}^{i}}{d s^{\mathbf{\alpha}}}+\bar{\Gamma}_{\alpha \beta}^{i} \frac{d \bar{x}^{\alpha}}{d s} \frac{d \bar{x}^{\beta}}{d s}=0
$$

in which

$$
\bar{\Gamma}_{j k}^{i}=\frac{\partial \bar{x}^{i}}{\partial x^{\alpha}}\left(\frac{\partial^{2} x^{\alpha}}{\partial \bar{x}^{j} \partial \bar{x}^{k}}+\Gamma_{\beta \gamma}^{\alpha} \frac{\partial x^{\beta}}{\partial \bar{x}^{j}} \frac{\partial x^{\gamma}}{\partial \bar{x}^{k}}\right) .
$$

Thus the form of the equation (2.1) persists under a transformation of coördinates. It follows from (3.7) that the runctions $\Gamma$ behave like the components of a tensor under linear transformations with constant coefficients but not under more general transformations. It is seen by an easy computation that the functions $B_{j k l}^{i}$ defined in $\S 2$ are the components of a tensor. It follows at once that the equation

$$
S_{k l}=B_{i k l}^{i}=\frac{\partial \Gamma_{i k}^{i}}{\partial x^{l}}-\frac{\partial \Gamma_{i l}^{i}}{\partial x^{k}}
$$

defines a tensor which is skew symmetric. This tensor is identically zero in the Riemann geometry. It also follows that

$$
R_{j k}=B_{j k i}^{i}=\frac{\partial \Gamma_{j k}^{i}}{\partial x^{i}}-\frac{\partial \Gamma_{j i}^{i}}{\partial x^{k}}+\Gamma_{j k}^{\alpha} \Gamma_{\alpha i}^{i}-\Gamma_{j i}^{\alpha} \Gamma_{\alpha k}^{i}
$$

is a tensor. This we shall call the Ricci tensor because it reduces to the tensor studied by Ricci* for the case of the Riemann geometry. It is symmetric if and only if $S_{i j}=0$, as is obvious on comparing (3.8) and (3.9). Further properties of these tensors are to be found in a paper by Eisenhart in the Annals of Mathematics (vol. 24).

For convenience of reference we put down here the following formulas about transformation of coordinates in general:

\footnotetext{
*G. Ricci, Atti, Reale Istitu to Veneto, vol. 63 (1903), pp. 1233-1239.
} 


$$
\frac{\partial \bar{x}^{i}}{\partial x^{\alpha}} \frac{\partial x^{\alpha}}{\partial \bar{x}^{j}}=\delta_{j}^{i}
$$

$$
\frac{\partial^{2} \bar{x}^{i}}{\partial x^{\alpha} \partial x^{\beta}} \frac{\partial x^{\alpha}}{\partial \bar{x}^{j}}+\frac{\partial^{2} x^{\alpha}}{\partial \bar{x}^{j} \partial \bar{x}^{k}} \frac{\partial \bar{x}^{i}}{\partial x^{\alpha}} \frac{\partial \bar{x}^{k}}{\partial x^{\beta}}=0
$$

$$
\frac{\partial^{2} \bar{x}^{i}}{\partial x^{\alpha} \partial x^{\beta}} \frac{\partial x^{\alpha}}{\partial \bar{x}^{j}} \frac{\partial x^{\beta}}{\partial \bar{x}^{k}}+\frac{\partial^{2} x^{\alpha}}{\partial \bar{x}^{j} \partial \bar{x}^{k}} \frac{\partial \bar{x}^{i}}{\partial x^{\alpha}}=0
$$

$$
\frac{\partial^{3} \bar{x}^{i}}{\partial x^{\alpha} \partial x^{\beta} \partial x^{\gamma}} \frac{\partial x^{\alpha}}{\partial \bar{x}^{j}}+\frac{\partial^{2} \bar{x}^{i}}{\partial x^{\alpha} \partial x^{\beta}} \frac{\partial^{2} x^{\alpha}}{\partial \bar{x}^{j} \partial \bar{x}^{k}} \frac{\partial \bar{x}^{k}}{\partial x^{\gamma}}+\frac{\partial^{8} x^{\alpha}}{\partial \bar{x}^{j} \partial \bar{x}^{k} \partial \bar{x}^{l}} \frac{\partial \bar{x}^{i}}{\partial x^{\alpha}} \frac{\partial \bar{x}^{k}}{\partial x^{\beta}} \frac{\partial \bar{x}^{l}}{\partial x^{\gamma}}
$$

$$
\begin{gathered}
+\frac{\partial^{2} x^{\alpha}}{\partial \bar{x}^{j} \partial \bar{x}^{k}} \frac{\partial^{2} \bar{x}^{i}}{\partial x^{\alpha} \partial x^{\gamma}} \frac{\partial \bar{x}^{k}}{\partial x^{\beta}}+\frac{\partial^{2} x^{\alpha}}{\partial \bar{x}^{j} \partial \bar{x}^{k}} \frac{\partial \bar{x}^{i}}{\partial x^{\alpha}} \frac{\partial^{2} \bar{x}^{k}}{\partial x^{\beta} \partial x^{\gamma}}=0 \\
\frac{\partial^{3} \bar{x}^{i}}{\partial x^{\alpha} \partial x^{\beta} \partial x^{\gamma}} \frac{\partial x^{\alpha}}{\partial \bar{x}^{j}} \frac{\partial x^{\gamma}}{\partial \bar{x}^{l}}+\frac{\partial^{2} \bar{x}^{i}}{\partial x^{\alpha} \partial x^{\beta}} \frac{\partial^{2} x^{\alpha}}{\partial \bar{x}^{j} \partial \bar{x}^{l}}+\frac{\partial^{8} x^{\alpha}}{\partial \bar{x}^{j} \partial \bar{x}^{k} \partial \bar{x}^{l}} \frac{\partial \bar{x}^{i}}{\partial x^{\alpha}} \frac{\partial \bar{x}^{k}}{\partial x^{\beta}}
\end{gathered}
$$

$$
+\frac{\partial^{2} x^{\alpha}}{\partial \bar{x}^{j} \partial \bar{x}^{k}} \frac{\partial^{2} \bar{x}^{i}}{\partial x^{\alpha} \partial x^{\gamma}} \frac{\partial \bar{x}^{k}}{\partial x^{\beta}} \frac{\partial x^{\gamma}}{\partial \bar{x}^{l}}+\frac{\partial^{2} x^{\alpha}}{\partial \bar{x}^{j} \partial \bar{x}^{k}} \frac{\partial^{2} \bar{x}^{k}}{\partial x^{\beta} \partial x^{\gamma}} \frac{\partial \bar{x}^{i}}{\partial x^{\alpha}} \frac{\partial x^{\gamma}}{\partial \bar{x}^{l}}=0 ;
$$

$$
\frac{\partial^{3} \bar{x}^{i}}{\partial x^{\alpha} \partial x^{\beta} \partial x^{\gamma}} \frac{\partial x^{\alpha}}{\partial \bar{x}^{j}} \frac{\partial x^{\beta}}{\partial \bar{x}^{k}}+\frac{\partial^{2} \bar{x}^{i}}{\partial x^{\alpha} \partial x^{\beta}} \frac{\partial^{2} x^{\alpha}}{\partial \bar{x}^{j} \partial \bar{x}^{l}} \frac{\partial x^{\beta}}{\partial \bar{x}^{k}} \frac{\partial \bar{x}^{l}}{\partial x^{\gamma}}
$$

$+\frac{\partial^{2} \bar{x}^{i}}{\partial x^{\alpha} \partial x^{\beta}} \frac{\partial^{2} x^{\beta}}{\partial \bar{x}^{k} \partial \bar{x}^{l}} \frac{\partial x^{\alpha}}{\partial \bar{x}^{j}} \frac{\partial \bar{x}^{l}}{\partial x^{\gamma}}+\frac{\partial^{3} x^{\alpha}}{\partial \bar{x}^{j} \partial \bar{x}^{k} \partial \bar{x}^{l}} \frac{\partial \bar{x}^{i}}{\partial x^{\alpha}} \frac{\partial \bar{x}^{l}}{\partial x^{\gamma}}+\frac{\partial^{2} x^{\alpha}}{\partial \bar{x}^{j} \partial \bar{x}^{k}} \frac{\partial^{2} \bar{x}^{i}}{\partial x^{\alpha} \partial x^{\gamma}}=0 ;$

$$
\frac{\partial^{3} \bar{x}^{i}}{\partial x^{\alpha} \partial x^{\beta} \partial x^{\gamma}} \frac{\partial x^{\alpha}}{\partial \bar{x}^{j}} \frac{\partial x^{\beta}}{\partial \bar{x}^{k}} \frac{\partial x^{\gamma}}{\partial \bar{x}^{l}}+\frac{\partial^{2} \bar{x}^{i}}{\partial x^{\alpha} \partial x^{\beta}} \frac{\partial^{2} x^{\alpha}}{\partial \bar{x}^{j} \partial \bar{x}^{l}} \frac{\partial x^{\beta}}{\partial \bar{x}^{k}}
$$

$$
+\frac{\partial^{2} \bar{x}^{i}}{\partial x^{\alpha} \partial x^{\beta}} \frac{\partial^{2} x^{\beta}}{\partial \bar{x}^{k} \partial \bar{x}^{l}} \frac{\partial x^{\alpha}}{\partial \bar{x}^{j}}+\frac{\partial^{3} x^{\alpha}}{\partial \bar{x}^{j}} \frac{\partial \bar{x}^{k} \partial \bar{x}^{l}}{\partial x^{\alpha}}+\frac{\partial^{2} x^{\alpha}}{\partial \bar{x}^{j} \partial \bar{x}^{k}} \frac{\partial^{2} \bar{x}^{i}}{\partial x^{\alpha} \partial x^{\beta}} \frac{\partial x^{\beta}}{\partial \bar{x}^{l}}=0 .
$$

4. Transformation of the independent variable. If we make an arbitrary analytic substitution

$$
\begin{aligned}
& s=f(t), \\
& t=g(s),
\end{aligned}
$$


in the equation of a path (2.3) the latter becomes

$$
x^{i}=\phi^{i}(t) .
$$

For this path we have

$$
\frac{d^{2} x^{i}}{d s^{2}}=\frac{d^{2} x^{i}}{d t^{2}}\left(\frac{d t}{d s}\right)^{2}+\frac{d x^{i}}{d t} \frac{d^{2} t}{d s^{2}} .
$$

On comparison with (2.1) we see that the equation (4.3) satisfies the differential equation

$$
\frac{\frac{d^{2} x^{i}}{d t^{2}}+I_{\alpha \beta}^{i} \frac{d x^{\alpha}}{d t} \frac{d x^{\beta}}{d t}}{\frac{d x^{i}}{d t}}=-\frac{\frac{d^{2} t}{d s^{2}}}{\left(\frac{d t}{d s}\right)^{2}} .
$$

Hence the differential equations

$$
\frac{\frac{d^{2} x^{i}}{d t^{2}}+\Gamma_{\alpha \beta}^{i} \frac{d x^{\alpha}}{d t} \frac{d x^{\beta}}{d t}}{\frac{d x^{i}}{d t}}=\frac{\frac{d^{2} x^{j}}{d t^{2}}+\Gamma_{\alpha \beta}^{j} \frac{d x^{\alpha}}{d t} \frac{d x^{\beta}}{d t}}{\frac{d x^{j}}{d t}}
$$

are satisfied by the equations of the paths and are such that they continue to be satisfied if the independent variable in the parameter representation (4.3) of any path is subjected to an arbitrary transformation.

From (4.5) it is evident that the differential equations (2.1) will continue to be satisfied if the independent variable in the equations of a path (2.3) be replaced by $t$ where

$$
t=a s+b,
$$

$a$ and $b$ being constants.

The differential equations (4.6) are due to J. L. Synge, who has pointed out that the system of paths defined by them is no more general than that defined by (2.1). For, suppose that (4.3) satisfies (4.6). Let $\Phi(t)$ be the function of $t$ obtained by substituting (4.3) in any of the expressions whose equality is asserted by (4.6). The following equation is satisfied by (4.3):

$$
\frac{\frac{d^{2} x^{i}}{d t^{2}}+\Gamma_{\alpha \beta}^{i} \frac{d x^{\alpha}}{d t} \frac{d x^{\beta}}{d t}}{\frac{d x^{i}}{d t}}=\boldsymbol{\Phi}(t) .
$$


Substitute (4.2) in (4.3), obtaining

$$
x^{i}=\psi(s),
$$

an equation of the paths which must satisfy

$$
\frac{\frac{d^{2} x^{i}}{d s^{2}}+\Gamma_{\alpha \beta}^{i} \frac{d x^{\alpha}}{d s} \frac{d x^{\beta}}{d s}}{\frac{d x^{i}}{d s}}=\frac{\Phi(t)}{\frac{d s}{d t}}-\frac{\frac{d^{2} s}{d t^{2}}}{\left(\frac{d s}{d t}\right)^{2}}
$$

Now if

$$
s=f(t)=A+B \int e^{\int d(t) d t} d t
$$

$A$ and $B$ being constants, (4.9) reduces to (2.1). Hence the equations of any path defined by (4.6) may be written as solutions of (2.1).

5. Projective geometry of paths.* Let us inquire under what circumstances a set of differential equations

$$
\frac{d^{2} x^{i}}{d s^{2}}+\Lambda_{\alpha \beta}^{i} \frac{d x^{\alpha}}{d s} \frac{d x^{\beta}}{d s}=0
$$

can represent the same system of paths as (2.1). Suppose that a curve

$$
x^{i}=\varphi^{i}(t)
$$

is a path both for (5.1) and.for (2.1). The functions $\varphi^{i}(t)$ are not necessarily solutions of (2.1) or of (5.1), but they are solutions of Synge's equations (4.6) and also of the corresponding equations determined by (5.1), i. e. of

(5.3) $\frac{d x^{j}}{d t}\left(\frac{d^{2} x^{i}}{d t^{2}}+A_{\alpha \beta}^{i} \frac{d x^{\alpha}}{d t} \frac{d x^{\beta}}{d t}\right)=\frac{d x^{i}}{d t}\left(\frac{d^{2} x^{j}}{d t^{2}}+\Lambda_{\alpha \beta}^{j} \frac{d x^{\alpha}}{d t} \frac{d x^{\beta}}{d t}\right)$.

* The discovery that the same system of paths arises from (5.1) as from (2.1) when (5.5) and (5.8) are satisfied is due to Weyl, Göttinger Nachrichten, 1921, p. 99. See also Eisenhart, Proceedings of the National Academy of Sciences, vol. 8 (1922), p. 233, and Veblen, ibid., p. 347. In the latter paper in equation (2.6) the final $t$ should be omitted and $d x^{1} / d t, \ldots, d x^{n} / d t$ should be evaluated at the point $q$; also the integration signs are missing in (4.2). 
Between (5.3) and (4.6) we can eliminate the second derivatives, thus obtaining

$$
\frac{\left(\Gamma_{\alpha \beta}^{i}-\Lambda_{\alpha \beta}^{i}\right)}{\frac{d x^{i}}{d t}} \frac{d x^{\alpha}}{d t} \frac{d x^{\beta}}{d t}=\frac{\left(\Gamma_{\alpha \beta}^{j}-\Lambda_{\alpha \beta}^{j}\right)}{\frac{d x^{j}}{d t}} \frac{d x^{\alpha}}{d t} \frac{d x^{\beta}}{d t} .
$$

Let

$$
\Gamma_{\alpha \beta}^{i}-\Lambda_{\alpha \beta}^{i}=\Phi_{\alpha \beta}^{i}
$$

and

$$
\frac{1}{n+1} \boldsymbol{\Phi}_{i \beta}^{i}=\Phi_{\beta}
$$

If we subtract from (3.7) the corresponding equations for the functions $A$ the result shows that $\Phi_{\alpha \beta}^{i}$ is a tensor. Hence $\Phi_{\beta}$ is a vector. The equation (5.4) now becomes

$$
\left(\Phi_{\alpha \beta}^{i} \frac{d x^{j}}{d t}-\Phi_{\alpha \beta}^{j} \frac{d x^{i}}{d t}\right) \frac{d x^{\alpha}}{d t} \frac{d x^{\beta}}{d t}=0
$$

In this we put

$$
\frac{d x^{i}}{d t}=\delta_{\gamma}^{i} \frac{d x^{\gamma}}{d t} \text { and } \frac{d x^{j}}{d t}=\delta_{\gamma}^{j} \frac{d x^{\gamma}}{d t}
$$

and obtain

$$
\left(\Phi_{\alpha \beta}^{i} \delta_{\gamma}^{j}-\Phi_{\alpha \beta}^{j} \delta_{\gamma}^{i}\right) \frac{d x^{\alpha}}{d t} \frac{d x^{\beta}}{d t} \frac{d x^{\gamma}}{d t}=0
$$

Since the derivatives $d x^{\alpha} / d t$ may be chosen arbitrarily this gives

$$
\Phi_{\alpha \beta}^{i} \delta_{\gamma}^{j}+\Phi_{\beta \gamma}^{i} \delta_{\alpha}^{j}+\Phi_{\gamma \alpha}^{i} \delta_{\beta}^{j}=\Phi_{\alpha \beta}^{j} \delta_{\gamma}^{i}+\Phi_{\beta \gamma}^{j} \delta_{\alpha}^{i}+\Phi_{\gamma \alpha}^{j} \delta_{\beta}^{i} .
$$

If we set $j=\gamma$ in this equation and sum with respect to $\gamma$, we obtain

$$
n \boldsymbol{\Phi}_{\alpha \beta}^{i}+\boldsymbol{\Phi}_{\beta \alpha}^{i}+\boldsymbol{\Phi}_{\beta \alpha}^{i}=\boldsymbol{\Phi}_{\alpha \beta}^{i}+(n+1) \boldsymbol{\Phi}_{\beta} \delta_{\alpha}^{i}+(n+1) \boldsymbol{\Phi}_{\alpha} \delta_{\beta}^{i} .
$$

Hence

$$
\Phi_{\alpha \beta}^{i}=\Phi_{\alpha} \delta_{\beta}^{i}+\Phi_{\beta} \delta_{\alpha}^{i}
$$

Hence, if the equations (5.1) and (2.1) are to determine the same system of paths, the functions $\Gamma$ and $A$ must be related by (5.5) and (5.8). 
Conversely, let $\Phi_{\alpha}$ be any covariant vector and let the tensor $\Phi_{\alpha \beta}^{i}$ be defined by the equations (5.8). Then any two sets of differential equations (2.1) and (5.1) will define the same system of paths, provided that (5.5) is satisfied. For consider any path with respect to the $\Gamma$ 's. Along this path we have

$$
2 \boldsymbol{\Phi}_{\alpha} \frac{d x^{\alpha}}{d t}=\frac{\boldsymbol{\Phi}_{\alpha \beta}^{i} \frac{d x^{\alpha}}{d t} \frac{d x^{\beta}}{d t}}{\frac{d x^{i}}{d t}}=\frac{\left(\Gamma_{\alpha \beta}^{i}-\lambda_{\alpha \beta}^{i}\right)}{\frac{d x^{i}}{d t}} \frac{d x^{\alpha}}{d t} \frac{d x^{\beta}}{d t} .
$$

Hence (5.4) is satisfied. But if (5.4) is added to (4.6) the corresponding equations in $\boldsymbol{A}$ are obtained. Hence every path with respect to the $\Gamma$ 's is also a path with respect to the $A$ 's.

A system of functions $\Gamma_{\alpha \beta}^{i}$ determines a definition of infinitesimal parallelism in the sense of Levi-Civita and Weyl. It is therefore appropriate to designate the body of theorems which state those properties which are determined by a particular set of differential equations (2.1) as an affine geometry of paths. In like manner the body of theorems which state properties of a system of paths independently of any particular definition of affine connection (i. e. of any particular set of differential equations (2.1)) may be called a projective geometry of paths.

For example, the theory of the curvature tensor belongs to the affine geometry of paths. For if the curvature tensor determined by (2.1) is denoted by $B_{\alpha \beta \gamma}^{i}$ as in $\S 2$, the corresponding curvature tensor determined by (5.1) is

$$
B_{\alpha, \beta \gamma}^{i}-\delta_{\alpha}^{i} \Phi_{\beta, \gamma}+\delta_{\alpha}^{i} \Phi_{\gamma, \beta}-\delta_{\beta}^{i} \Phi_{\alpha, \gamma}+\delta_{\gamma}^{i} \Phi_{\alpha, \beta}-\delta_{\beta}^{i} \Phi_{\alpha} \Phi_{\gamma}+\delta_{\gamma}^{i} \Phi_{\alpha} \Phi_{\beta}
$$

In this expression $\Phi_{\alpha, \beta}$ denotes the covariant derivative (cf. $\S 10$ below) of $\Phi_{\alpha}$ with respect to the functions $\Gamma_{\alpha \beta}^{i}$.

The Ricci tensor $R_{\alpha \beta}$ becomes

$$
R_{\alpha \beta}+n \Phi_{\alpha, \beta}-\Phi_{\beta, \alpha}+(n-1) \Phi_{\alpha} \Phi_{\beta},
$$

and the skew symmetric tensor $S_{\alpha \beta}$ becomes

$$
S_{\alpha \beta}-(n+1)\left(\Phi_{\alpha, \beta}-\Phi_{\beta, \alpha}\right)
$$


Comparing these three expressions, it is evident that a tensor which is the same for the $A$ 's as for the $\Gamma$ 's is defined as follows:

$$
W_{\alpha \beta \gamma}^{i}=B_{\alpha \beta \gamma}^{i}-\frac{\delta_{\gamma}^{i} R_{\alpha \beta}}{n-1}+\frac{\delta_{\beta}^{i} R_{\alpha \gamma}}{n-1}-\frac{1}{n^{2}-1}\left(\delta_{\gamma}^{i} S_{\alpha \beta}-\delta_{\beta}^{i} S_{\alpha \gamma}\right)-\frac{\delta_{\alpha}^{i} S_{\beta \gamma}}{n+1}
$$

This is what Weyl (loc. cit.) calls the projective curvature tensor, and its theory belongs to the projective geometry of paths. It can also be written in the form

$$
\begin{aligned}
W_{\alpha \beta \gamma}^{i}=B_{\alpha \beta \gamma}^{i}+\frac{\delta_{\beta}^{i}}{n^{2}-1} & \left(n R_{\alpha \gamma}+R_{\gamma \alpha}\right) \\
& -\frac{\delta_{\gamma}^{i}}{n^{2}-1}\left(n R_{\alpha \beta}+R_{\beta \alpha}\right)+\frac{\delta_{\alpha}^{i}}{n+1}\left(R_{\beta \gamma}-R_{\gamma \beta}\right) .
\end{aligned}
$$

In the rest of this paper we shall be concerned entirely with the affine geometry of paths, to which we now return.

6. Equations of the paths. A unique solution of (2.1) in the form (2.3) can, be found which satisfies a set of initial conditions

$$
\begin{aligned}
q^{i} & =\psi^{i}(0) \\
\xi^{i} & =\frac{d}{d s} \psi^{i}(0)
\end{aligned}
$$

where $q^{1}, q^{2}, \ldots, q^{n}$ and $\xi^{1}, \xi^{\mathbf{y}}, \ldots, \xi^{n}$ are arbitrary constants. For if we differentiate (2.1) successively we obtain the following sequence of equations:

$$
\begin{aligned}
& \frac{d^{2} x^{i}}{d s^{2}}+\Gamma_{\alpha \beta}^{i} \frac{d x^{\alpha}}{d s} \frac{d x^{\beta}}{d s}=0, \\
& \frac{d^{3} x^{i}}{d s^{3}}+\Gamma_{\alpha \beta \gamma}^{i} \frac{d x^{\alpha}}{d s} \frac{d x^{\beta}}{d s} \frac{d x^{\gamma}}{d s}=0, \\
& \frac{d^{4} x^{i}}{d s^{4}}+\Gamma_{\alpha \beta \gamma \delta}^{i} \frac{d x^{\alpha}}{d s} \frac{d x^{\beta}}{d s} \frac{d x^{\gamma}}{d s} \frac{d x^{\delta}}{d s}=0,
\end{aligned}
$$


in which

$$
\Gamma_{j k l}^{i}=\frac{1}{3} P\left(\frac{\partial \Gamma_{j k}^{i}}{\partial x^{l}}-\Gamma_{\alpha k}^{i} \Gamma_{j l}^{\alpha}-\Gamma_{j k}^{i} \Gamma_{k l}^{\alpha}\right)=\frac{1}{3} P\left(\frac{\partial \Gamma_{j k}^{i}}{\partial x^{l}}-2 \Gamma_{\alpha j}^{i} \Gamma_{k l}^{\alpha}\right)
$$

and, in general,

$$
\begin{aligned}
\Gamma_{j k l \ldots m n}^{i}=-\frac{1}{N} P\left(\frac{\partial \Gamma_{j k l}^{i} \ldots m}{\partial x^{n}}\right. & \left.-\Gamma_{\alpha k l \ldots m}^{i} \Gamma_{j n}^{\alpha}-\ldots-\Gamma_{j k l \ldots \alpha}^{e} \Gamma_{m n}^{\alpha}\right) \\
= & \frac{1}{N} P\left[\frac{\partial \Gamma_{j k l \ldots m}^{i} \ldots m}{\partial x^{n}}-(N-1) \Gamma_{a j k \ldots l}^{i} \Gamma_{m n}^{\alpha}\right]
\end{aligned}
$$

where $N$ denotes the number of subscripts, and the symbol $P$ denotes the sum of the terms obtainable from the ones inside the parenthesis by permuting the set of subscripts cyclically. Thus the functions $\Gamma_{j k l \ldots m n}^{i}$ have the property of being unchanged by any permutation of the subscripts.* The equations (6.1), (6.2), (6.3) determine immediately the following series for $\psi^{i}$ in terms of $s$ :

$$
x^{i}=q^{i}+\xi^{i} s-\frac{1}{2 !} \Gamma_{\alpha \beta}^{i}(q) \xi^{\alpha} \xi^{\beta} s^{2}-\frac{1}{3 !} \Gamma_{\alpha \beta \gamma}^{i}(q) \xi^{\alpha} \xi^{\beta} \xi^{\gamma} s^{3}-\cdots
$$

In this expression $\Gamma_{\alpha \beta \ldots \mu}^{i}(q)$ represents the value of $\Gamma_{\alpha \beta \ldots \mu}^{i}$ obtained by giving $x^{i}$ the value $q^{i}$. In general we shall use $x$ to represent $\left(x^{1}, x^{2}, \ldots, x^{n}\right)$, $\xi$ to represent $\left(\xi^{1}, \xi^{2}, \ldots, \xi^{n}\right)$, and so on. For any point $q$ and any "direction" $\xi$ we have a unique path determined by (6.6). These equations may be abbreviated in the form

$$
x^{i}=q^{i}+\Gamma^{i}(q, \xi s)
$$

The jacobian of the equations (6.7) is equal to unity. Hence for values of $x$ sufficiently near to $q$ the equations can be solved, giving

$$
\xi^{i} s=x^{i}-q^{i}+A^{i}(q, x-q),
$$

where $\boldsymbol{A}^{i}$ is a multiple power series in $\left(x^{i}-q^{i}\right)$, beginning with second order terms. Hence there is one and only one path joining $q$ to $x$.

* We have changed the notation used by Veblen in the Proceedings of the National Academy of Sciences, vol. 8 (1922), p. 192, in order to introduce this symmetry. 
7. Normal coördinates. Let us now put

$$
y^{i}=\xi^{i} s
$$

The equations (6.7) and (6.8) become

$$
x^{i}=q^{i}+\Gamma^{i}(q, y)
$$

and

$$
y^{i}=x^{i}-q^{i}+A^{i}(q, x-q)
$$

These equations may be regarded as defining a transformation from the coördinates $\left(x^{1}, x^{2}, \ldots, x^{n}\right)$ to a new set of coorrdinates $\left(y^{1}, y^{2}, \ldots, y^{n}\right)$ which we shall call normal coördinates because they reduce to Riemann's normal coördinates in case the geometry of paths reduces to a Riemann geometry. This transformation changes the differential equations of the paths (2.1) into

$$
\frac{d^{2} y^{i}}{d s^{2}}+C_{\alpha \beta}^{i} \frac{d y^{\alpha}}{d s} \frac{d y^{\beta}}{d s}=0
$$

where $C_{\alpha \beta}^{i}$ are functions of $y$ defined by the equations

$$
C_{j k}^{\gamma \alpha} \frac{\partial x^{i}}{\partial y^{\alpha}}=-\frac{\partial^{2} x^{i}}{\partial y^{j} \partial y^{k}}+\Gamma_{\beta \gamma}^{i} \frac{\partial x^{\beta}}{\partial y^{j}} \frac{\partial x \gamma}{\partial y^{k}}
$$

These coorrdinates have been so chosen that the curves defined by (7.1) are the paths through the origin. If we take any point $y$ there is one and only one of the paths (7.1) which passes through it. Substituting (7.1) in (7.4) we find

$$
C_{\alpha \beta}^{i} \dot{\xi}^{\alpha} \xi^{\beta}=0
$$

and hence on multiplying by the square of the value of $s$ determined for the point $y$ by the equation (7.1) we obtain

$$
C_{\alpha \beta}^{i} y^{\alpha} y^{\beta}=0
$$

Let us now consider the effect of a transformation of the variables $x$ of the form (3.1). This changes the equation of a path (2.3) which satisfies (2.1) 
into the equation (3.3) which satisfies (3.6). It also changes the initial conditions (6.1) and (6.2) into

$$
\bar{q}^{i}=\bar{\Psi}^{i}(0)=f^{i}(q)
$$

and

$$
\bar{\xi}^{i}=\frac{d}{d s} \bar{\Psi}^{i}(0)=\frac{d \psi^{\alpha}(0)}{d s}\left(\frac{\partial \bar{x}^{i}}{\partial x^{\alpha}}\right)_{q}
$$

respectively, the subscript $q$ indicating that the derivative is evaluated for $x=q$. For any point $p$, not too far away from $q$, there is a unique path and thus a unique set of values $\left(y^{1}, y^{2}, \ldots, y^{n}\right)$. From these we determine $\xi^{i}$ and $s$ so that $y^{i}=\xi^{i} s$. Then (3.3) gives the equation of the same path in terms of the coordinates $\bar{x}$ in such form that the point $p$ is determined by the parameter $s$. Hence by (7.9),

$$
\bar{y}^{i}=y^{\alpha}\left(\frac{\partial \bar{x}^{i}}{\partial x^{\alpha}}\right)_{q}
$$

In this formula the coefficients $\left(\frac{\partial \bar{x}^{i}}{\partial x^{\alpha}}\right)_{q}$ are independent of the particular path and dependent only on the point $q$ and the two coördinate systems. Hence when the coördinates $x$ undergo an arbitrary analytic transformation, the normal coördinates determined by the coördinates $x$ and a point $q$ suffer a linear homogeneous transformation (7.10) with constant coefficients. In other words the normal coördinates are transformed like contravariant vectors. They are not vectors, however, in the narrow sense, but are the components of a "step" from the origin of the normal coordinates to the point at which the coorrdinates are taken. An arbitrary step $(A B)$ determined by the points $A$ and $B$ can be represented by the coordinates of the point $B$ in the normal coordinate system associated with the point $A$.

8. Alternative treatment of normal coördinates. The identity (7.7) can be used as the definition of the normal coördinates. For by (3.12)

$$
C_{\alpha \beta}^{i}=\left(\Gamma_{j k}^{\gamma} \frac{\partial y^{i}}{\partial x^{\gamma}}-\frac{\partial^{y} y^{i}}{\partial x^{j} \partial x^{k}}\right) \frac{\partial x^{j}}{\partial y^{\alpha}} \frac{\partial x^{k}}{\partial y^{\beta}}
$$

so that (7.7) becomes

$$
\left(\Gamma_{j k}^{\gamma} \frac{\partial y^{i}}{\partial x^{\gamma}}-\frac{\partial^{2} y^{i}}{\partial x^{j} \partial x^{k}}\right) \frac{\partial x^{j}}{\partial y^{\alpha}} \frac{\partial x^{k}}{\partial y^{\beta}} y^{\alpha} y^{\beta}=0 .
$$


The differential equations (8.2) uniquely determine a functional relation between the $x$ 's and the $y$ 's when taken in conjunction with the initial conditions

$$
\begin{aligned}
y^{i} & =0 \text { when } x^{i}=q^{i}, \\
\frac{\partial y^{i}}{\partial x^{j}} & =\delta_{j}^{i} \text { when } x^{i}=q^{i} .
\end{aligned}
$$

For when we differentiate (8.2) repeatedly and substitute these initial conditions, making use of the formulas at the end of $\S 3$, we find

$$
x^{i}=q^{i}+y^{i}-\frac{1}{2 !} \Gamma_{\alpha \beta}^{i}(q) y^{\alpha} y^{\beta}-\frac{1}{3 !} \Gamma_{\alpha \beta \gamma}^{i}(q) y^{\alpha} y^{\beta} y^{\gamma}
$$

$$
-\frac{1}{4 !} \Gamma_{\alpha \beta \gamma \delta}^{i} y^{\alpha} y^{\beta} y^{\gamma} y^{\delta}-\cdots
$$

and

$$
y^{i}=x^{i}-q^{i}+\frac{1}{2 !} \lambda_{\alpha \beta}^{i}(q)\left(x^{\alpha}-q^{\alpha}\right)\left(x^{\beta}-q^{\beta}\right)
$$

$$
+\frac{1}{3 !} A_{\alpha \beta \gamma}^{i}\left(x^{\alpha}-q^{\alpha}\right)\left(x^{\beta}-q^{\beta}\right)\left(x^{\gamma}-q^{\gamma}\right)+\cdots
$$

where the $\Gamma$ 's have the meaning given them in $\S 3$ and the $A$ 's are such that

$$
\begin{aligned}
\Lambda_{j k}^{i} & =\Gamma_{j k,}^{i}, \\
\Lambda_{j k l}^{i} & =\Gamma_{j k l}^{i}+P\left(\Lambda_{\alpha j}^{i} \Gamma_{k l}^{c}\right)=\frac{1}{3} P\left(\frac{\partial \Gamma_{j k}^{i}}{\partial x^{l}}-\Gamma_{\alpha j}^{i} \Gamma_{k l}^{\alpha}\right), \\
\Lambda_{j k l m}^{i} & =\Gamma_{j k l m}^{i}+P\left(\Lambda_{\alpha j}^{i} \Gamma_{k l m}^{\alpha}+\Lambda_{\alpha \beta}^{i} I_{j k}^{\alpha} \Gamma_{l m}^{\beta}+\Lambda_{\alpha j k}^{i} \Gamma_{l m}^{\alpha}\right),
\end{aligned}
$$

If the general solution of (8.2), regarded as a differential equation for $y$ in terms of $x$, is denoted by $\tilde{y}$ when only the initial conditions (8.3) are imposed, then

$$
\tilde{y}^{i}=a_{\alpha}^{i} y^{\alpha}
$$


where the $a_{\alpha}^{i}$ are arhitrary constants, and $y$ is the solution determined by the initial conditions (8.3) and (8.4) which is given by (8.6). This last theorem is proved by observing, first, that the function $\tilde{y}$ defined by (8.7) satisfies (8.2) and (8.3) and, second, that if there were any other solution for which

$$
\tilde{y}^{i}=0 \text { and } \frac{\partial \tilde{y}^{i}}{\partial x^{j}}=a_{j}^{i}
$$

when $x^{i}=q^{i}$, the solution (8.6) would not be uniquely determined by (8.4).

In order to show the tensor character of the normal corrdinates let us now consider the effect of a transformation of the variables $x$ of the form (3.1). We inquire what are the normal corrdinates determined by $\bar{x}^{1}, \bar{x}^{2}, \ldots, \bar{x}^{n}$. These normal coordinates which we shall denote by $\bar{y}^{1}, \bar{y}^{2}, \ldots, \bar{y}^{n}$ are solutions of

$$
\left(\bar{\Gamma}_{j k}^{p} \frac{\partial \bar{y}^{i}}{\partial \bar{x}^{p}}-\frac{\partial^{2} \bar{y}^{i}}{\partial \bar{x}^{j} \partial \bar{x}^{k}}\right) \frac{\partial \bar{x}^{j}}{\partial \bar{y}^{a}} \frac{\partial \bar{x}^{k}}{\partial \bar{y}^{\beta}} \bar{y}^{a} \bar{y}^{\beta}=0 .
$$

If we substitute into this the value of $\bar{\Gamma}_{j k}^{p}$ from (3.7) we obtain

$\left[\left(\Gamma_{q r}^{t} \cdot \frac{\partial x^{q}}{\partial \bar{x}^{j}} \frac{\partial x^{p}}{\partial \bar{x}^{k}} \frac{\partial \bar{x}^{p}}{\partial x^{t}}+\frac{\partial^{2} x^{t}}{\partial \bar{x}^{j} \partial \bar{x}^{k}} \frac{\partial \bar{x}^{p}}{\partial x^{t}}\right) \frac{\partial \bar{y}^{i}}{\partial \bar{x}^{p}}-\frac{\partial^{q} \bar{y}^{i}}{\partial \bar{x}^{j} \partial \bar{x}^{k}}\right] \frac{\partial \bar{x}^{j}}{\partial \bar{y}^{\alpha}} \frac{\partial \bar{x}^{k}}{\partial \bar{y}^{\beta}} \bar{y}^{\alpha} \bar{y}^{\beta}=0$,

or

$$
\left(\boldsymbol{\Gamma}_{q r}^{t} \frac{\partial \bar{y}^{i}}{\partial x^{t}}-\frac{\partial^{q} \bar{y}^{i}}{\partial x^{q} \partial x^{r}}\right) \frac{\partial x^{q}}{\partial \bar{y}^{\alpha}} \frac{\partial x^{r}}{\partial \bar{y}^{\beta}} \bar{y}^{\alpha} \bar{y}^{\beta}
$$

$$
+\left(\frac{\partial^{2} x^{t}}{\partial \bar{x}^{j} \partial \bar{x}^{k}} \frac{\partial \bar{y}^{i}}{\partial x^{t}}-\frac{\partial^{2} \bar{y}^{i}}{\partial \bar{x}^{j} \partial \bar{x}^{k}}+\frac{\partial^{2} \bar{y}^{i}}{\partial x^{q} \partial x^{\nu^{j}}} \frac{\partial x^{q}}{\partial \bar{x}^{j}} \frac{\partial x^{r}}{\partial \bar{x}^{k}}\right) \frac{\partial \bar{x}^{j}}{\partial \bar{y}^{\alpha}} \frac{\partial \bar{x}^{k}}{\partial \bar{y}^{\beta}} \bar{y}^{\alpha} \bar{y}^{\beta}=0 .
$$

The parenthesis in the second term is identically zero. Hence (8.9) is the same differential equation as (8.2).

By definition the normal coördinates must satisfy the initial conditions

$$
\bar{y}^{i}=0 \text { when } \bar{x}^{i}=\bar{q}^{i}
$$

and

$$
\delta_{j}^{i}=\frac{\partial \bar{y}^{i}}{\partial \bar{x}^{j}} \text { when } \bar{x}^{i}=\bar{q}^{i}
$$


Hence we must have

$$
\frac{\partial \bar{y}^{i}}{\partial x^{j}}=\frac{\partial \bar{x}^{i}}{\partial x^{j}} \text { when } \bar{x}^{i}=\bar{g}^{i}
$$

The value of $\partial \bar{x}^{i} / \partial x^{j}$ when $\bar{x}^{i}=\bar{q}^{i}$ is determined by (3.1). Let us call it $a_{j}^{i}$. Then by the theorem regarding the formula (8.7),

$$
\bar{y}^{i}=a_{\alpha}^{i} y^{\alpha}
$$

is a solution of (8.9) determined by the conditions

$$
\bar{y}^{i}=0 \quad \text { and } \quad \frac{\partial \bar{y}^{i}}{\partial \bar{x}^{j}}=\delta_{j}^{i} \quad \text { when } x^{i}=q^{i}
$$

Hence when the coordinates $x$ undergo an arbitrary transformation (3:1) the normal coordinates undergo a linear transformation (8.10) the coefficients $r i$ which are given by

$$
\frac{\partial f^{i}(q)}{\partial x^{j}}=a_{j}^{i}
$$

9. The normal tensors. Since $C_{j k}^{i}$ is symmetric in $j$ and $k$ and $\xi^{i}$ is entirely arbitrary it follows from (7.6) that $C_{j k}^{i}$ vanishes at the origin of normal coordinates, i. e.,

$$
\left(C_{j k}^{i}\right)_{0}=0
$$

Hence the power series for $C_{j k}^{i}$ takes the form

$$
C_{j k}^{i}=A_{j k \alpha}^{i} y^{\alpha}+\frac{1}{2 !} A_{j k \alpha \beta}^{i} y^{\alpha} y^{\beta}+\frac{1}{3 !} A_{j k \alpha \beta \gamma}^{i} y^{\alpha} y^{\beta} y^{\gamma}+\cdots
$$

in which the $A$ 's are the derivatives of $C_{j k}^{i}$ evaluated at the origin, i. e.,

$$
A_{j k \alpha}^{i} \ldots \tau=\left(\frac{\partial^{n} C_{j k}^{i}}{\partial y^{\alpha} \cdots \partial y^{\tau}}\right)_{0}
$$

The equation (9.3) can be taken as defining $A_{j k \alpha \ldots \tau}^{i}$ as a set of functions of $\left(x^{1}, x^{2}, \ldots, x^{n}\right)$. At any point $\left(p^{1}, p^{2}, \ldots, p^{n}\right), A_{j k \alpha \ldots \tau}^{i}$ is equal to the right 
hand member of (9.3) evaluated in the system of normal coordinates having $\left(p^{1}, p^{2}, \ldots, p^{n}\right)$ as origin. The functions so defined are tensors. For consider a transformation from $x$ to $\bar{x}$ and the transformation which it produces from $y$ to $\bar{y}$. By (3.7) we have

$$
\bar{C}_{j k}^{\alpha} \frac{\partial y^{i}}{\partial \bar{y}^{\alpha}}=C_{\beta \gamma}^{i} \frac{\partial y^{\beta}}{\partial \bar{y}^{j}} \frac{\partial y^{\gamma}}{\partial \bar{y}^{k}}
$$

and from (9.2)

$C_{\beta \gamma}^{i} \frac{\partial y^{\beta}}{\partial \bar{y}^{j}} \frac{\partial y^{\gamma}}{\partial \bar{y}^{k}}=A_{\beta \gamma \delta}^{i} \frac{\partial y^{\beta}}{\partial \bar{y}^{j}} \frac{\partial y^{\gamma}}{\partial \bar{y}^{k}} y^{\delta}+\frac{1}{2 !} A_{\beta \gamma \delta \varepsilon}^{i} \frac{\partial y^{\beta}}{\partial \bar{y}^{j}} \frac{\partial y^{\gamma}}{\partial \bar{y}^{k}} y^{\delta} y^{\varepsilon}+\cdots$

Hence

$$
\begin{aligned}
\bar{C}_{j k}^{\alpha} \frac{\partial y^{i}}{\partial \bar{y}^{\alpha}}=A_{\beta \gamma \delta}^{i} \frac{\partial y^{\beta}}{\partial \bar{y}^{j}} & \frac{\partial y^{\gamma}}{\partial \bar{y}^{k}} \frac{\partial y^{\delta}}{\partial \bar{y}^{\mu}} \bar{y}^{\mu} \\
& +\frac{1}{2 !} A_{\beta \gamma \delta \varepsilon}^{i} \frac{\partial y^{\beta}}{\partial \bar{y}^{j}} \frac{\partial y^{\gamma}}{\partial \bar{y}^{k}} \frac{\partial y^{\delta}}{\partial \bar{y}^{\mu}} \frac{\partial y^{\varepsilon}}{\partial \bar{y}^{\nu}} \bar{y}^{\mu} \bar{y}^{\nu}+\cdots
\end{aligned}
$$

Comparing this with the equation

$$
\bar{C}_{j k}^{i}=\bar{A}_{j k \mu}^{i} \bar{y}^{\mu}+\frac{1}{2 !} \bar{A}_{j k \mu \nu}^{i} \bar{y}^{\mu} \bar{y}^{\nu}+\cdots
$$

we have

$$
\bar{A}_{j k l \ldots m}^{\alpha} \frac{\partial y^{i}}{\partial \bar{y}^{\alpha}}=A_{\beta \gamma \delta \ldots \varepsilon}^{\alpha} \frac{\partial y^{\beta}}{\partial \bar{y}^{j}} \frac{\partial y^{\gamma}}{\partial \bar{y}^{k}} \frac{\partial y^{\delta}}{\partial \bar{y}^{l}} \cdots \frac{\partial y^{\varepsilon}}{\partial \bar{y}^{m}}
$$

If we make the substitution

$$
\frac{\partial y^{i}}{\partial \bar{y}^{j}}=\left(\frac{\partial x^{i}}{\partial \bar{x}^{j}}\right)_{0}
$$

then

$$
\bar{A}_{j k l \ldots m}^{\alpha} \frac{\partial x^{i}}{\partial \bar{x}^{\alpha}}=A_{\beta \gamma \delta \ldots \varepsilon}^{\alpha} \frac{\partial x^{\beta}}{\partial \bar{x}^{j}} \frac{\partial x^{\gamma}}{\partial \bar{x}^{k}} \frac{\partial x^{\delta}}{\partial \bar{x}^{l}} \cdots \frac{\partial x^{\varepsilon}}{\partial \bar{x}^{m}}
$$

where $A$ is regarded as a function of $\left(x^{1}, x^{2}, \ldots, x^{n}\right)$ and $\bar{A}$ as a function of $\left(\bar{x}^{1}, \bar{x}^{2}, \ldots, \bar{x}^{n}\right)$. This shows that $A_{j k l \ldots m}^{i}$ is a tensor which is contravariant in $i$ and covariant in $j k l \cdots m$. We shall call it a normal tensor because of its definition in terms of normal coordinates. 
By their definition (cf. (9.3)) these tensors are symmetric in the first two subscripts and also in the remaining ones, i. e.,

$$
\begin{aligned}
& A_{j k \alpha \beta \ldots \tau}^{i}=A_{k j \alpha \beta \ldots \tau}^{i}, \\
& A_{j k \alpha \beta \ldots \tau}^{i}=A_{j k \gamma \delta \ldots \sigma}^{i},
\end{aligned}
$$

where $\gamma \delta \ldots \sigma$ is intended to represent any permutation of $\alpha \beta \ldots \tau$.

If we multiply (9.2) by $y^{j} y^{k}$ and sum, the left member is zero by (7.7) and the right member is a multiple power series the coefficient of each term of which must be zero. It therefore follows that

$$
\begin{gathered}
A_{j k \alpha}^{i}+A_{k \alpha j}^{i}+A_{\alpha j k}^{i}=0, \\
A_{j k \alpha \beta}^{i}+A_{j \alpha k \beta}^{i}+A_{j \beta \alpha k}^{i}+A_{k \alpha j \beta}^{i}+A_{k \beta j \alpha}^{i}+A_{\alpha \beta j k}^{i}=0,
\end{gathered}
$$

and in general

$$
S\left(A_{j k \kappa \beta \ldots \tau}^{i}\right)=0
$$

where $S(\quad)$ stands for the sum of the $N(N-1) / 2$ terms obtainable from the one in the parenthesis and not identical because of (9.7) and (9.8).

The tensors $A$ are expressible in terms of the functions $\Gamma_{j k}^{i}$ and their derivatives. If we differentiate (7.5) we obtain

$$
\frac{\partial C_{j k}^{\alpha}}{\partial y^{l}} \frac{\partial x^{i}}{\partial y^{\alpha}}+C_{j k}^{\alpha} \frac{\partial^{2} x^{i}}{\partial y^{\alpha} \partial y^{l}}=\frac{\partial^{s} x^{i}}{\partial y^{j} \partial y^{k}} \frac{\partial y^{l}}{}
$$

$$
+\frac{\partial \Gamma_{\beta \gamma}^{i}}{\partial x^{\delta}} \frac{\partial x^{\beta}}{\partial y^{j}} \frac{\partial x^{\gamma}}{\partial y^{k}} \frac{\partial x^{\delta}}{\partial y^{l}}+\Gamma_{\beta \gamma}^{i} \frac{\partial^{2} x^{\beta}}{\partial y^{j}} \partial y^{i} \frac{\partial x^{\gamma}}{\partial y^{k}}+\Gamma_{\beta \gamma}^{i} \frac{\partial x^{\beta}}{\partial y^{j}} \frac{\partial^{2} x^{\gamma}}{\partial y^{k} \partial y^{l}} .
$$

Substituting the values of the partial derivatives of $x$ with regard to the $y$ 's as computed from (6.6) or (8.5) for the origin of normal coordinates, we find

$$
A_{j k l}^{i}=\frac{\partial \Gamma_{j k}^{i}}{\partial x^{l}}-\Gamma_{j k l}^{i}-\Gamma_{\beta k}^{i} \Gamma_{j l}^{\beta}-\Gamma_{j \beta}^{i} \Gamma_{k l}^{\beta}
$$


If we differentiate (9.12) again we obtain

$$
\begin{aligned}
A_{j k l m}^{i}= & \frac{\partial^{2} \Gamma_{j k}^{i}}{\partial x^{l} \partial x^{m}}-\Gamma_{j k l m}^{i}-\frac{\partial \Gamma_{j \beta}^{i}}{\partial x^{l}} \Gamma_{k m}^{\beta}-\frac{\partial \Gamma_{\beta k}^{i}}{\partial x^{l}} \Gamma_{j m}^{\beta}-\frac{\partial \Gamma_{j \beta}^{i}}{\partial x^{m}} \Gamma_{k l}^{\beta} \\
& -\frac{\partial \Gamma_{\beta k}^{i}}{\partial x^{m}} \Gamma_{j l}^{\beta}-\frac{\partial \Gamma_{j k}^{i}}{\partial x^{\beta}} \Gamma_{l m}^{\beta}-\Gamma_{j \beta}^{i} \Gamma_{k l m}^{\beta}-\Gamma_{\beta l}^{i} \Gamma_{j l m}^{\beta} \\
& +A_{j k l}^{\beta} \Gamma_{\beta m}^{i}+A_{j k m}^{\beta} \boldsymbol{I}_{\beta l}^{i}+\Gamma_{\beta \gamma}^{i} \Gamma_{j l}^{\beta} \Gamma_{k m}^{\gamma}+\Gamma_{\beta \gamma}^{i} \Gamma_{j m}^{\beta} \Gamma_{k l}^{\gamma}
\end{aligned}
$$

It is evident that a continuation of this process will determine the explicit formulas for any number of the $A$ 's.

10. Covariant differentiation. Covariant differentiation is a process by which from a given tensor there may be formed a new tensor with one more covariant index. Let $T_{i j \ldots k}^{l m_{\ldots} \ldots{ }^{n}}$ be any tensor referred to arbitrary coördinates $\left(x^{1}, x^{2}, \ldots, x^{n}\right)$ which is contravariant in $(l, m, \ldots, n)$ and covariant in $(i, j, \ldots, k)$. Let $t_{i j \ldots k}^{l m \ldots}{ }^{n}$ be the components of $T_{i j \ldots k}^{l m \ldots}$ in the normal coördinate system $\left(y^{1}, y^{2}, \ldots, y^{n}\right)$ which is determined by the $x$-coordinate system at the point $\left(q^{1}, q^{2}, \ldots, q^{n}\right)$. The equation

$$
T_{i j \ldots k, p}^{l m \ldots n}=\left(\frac{\partial t_{i j \ldots i}^{l m \ldots}{ }^{n}}{\partial y^{p}}\right)_{0}
$$

defines a set of functions of $x$ which turn out to be the components of a tensor. In the Riemann geometry this tensor is the same as the covariant derivative of $T$ according to the definition of Ricci and Levi-Civita. Hence we shall call it by the same name in the general case. The subscripts arising by covariant differentiation will be separated from those originally present in the tensor by a comma.

Let us now prove that the functions $T_{i j \ldots k, p}^{l m \ldots n}$ actually are the components of a tensor. Let the functions $T$ and $t$ become $\bar{T}$ and $\bar{t}$ respectively under the arbitrary transformation (3.1). This gives the equations

$$
\begin{aligned}
& \bar{T}_{i j \ldots k}^{l m \ldots n}=T_{\alpha \beta \ldots \gamma}^{\delta \varepsilon \ldots \nu} \frac{\partial \bar{x}^{l}}{\partial x^{\delta}} \frac{\partial \bar{x}^{m}}{\partial x^{\varepsilon}} \cdots \frac{\partial \bar{x}^{n}}{\partial x^{\nu}} \frac{\partial x^{\alpha}}{\partial \bar{x}^{i}} \frac{\partial x^{\beta}}{\partial \bar{x}^{j}} \cdots \frac{\partial x^{\gamma}}{\partial \bar{x}^{k}} \\
& \bar{t}_{i j \ldots k}^{l m \ldots n}=t_{\alpha \beta \ldots \gamma}^{\delta \varepsilon \ldots \nu} \frac{\partial \bar{y}^{l}}{\partial y^{\delta}} \frac{\partial \bar{y}^{m}}{\partial y^{\varepsilon}} \cdots \frac{\partial \bar{y}^{n}}{\partial y^{\nu}} \frac{\partial y^{\alpha}}{\partial \bar{y}^{i}} \frac{\partial y^{\beta}}{\partial \bar{y}^{j}} \cdots \frac{\partial y^{\gamma}}{\partial \bar{y}^{k}} .
\end{aligned}
$$


Since the derivatives in (10.3) are constants we obtain by partial differentiation (10.4) $\frac{\partial \bar{t}_{i j \ldots i^{n}}^{l m \ldots}}{\partial \bar{y}^{p}}=\frac{\partial t_{\alpha \beta \ldots \nu}^{\delta \varepsilon \ldots \nu}}{\partial y^{\omega}} \frac{\partial \bar{y}^{l}}{\partial y^{\delta}} \frac{\partial \bar{y}^{m}}{\partial y^{\varepsilon}} \cdots \frac{\partial \bar{y}^{n}}{\partial y^{\nu}} \frac{\partial y^{\alpha}}{\partial \bar{y}^{i}} \frac{\partial y^{\beta}}{\partial \bar{y}^{j}} \cdots \frac{\partial y^{\gamma}}{\partial \bar{y}^{k}} \frac{\partial y^{\omega}}{\partial \bar{y}^{p}}$, and, hence, at the point $q$ we have (10.5) $\bar{T}_{i j \ldots \ldots, p}^{l m \ldots n}=T_{\alpha \beta \ldots \gamma, \omega}^{\delta \varepsilon \ldots \nu} \frac{\partial \bar{x}^{l}}{\partial x^{\delta}} \frac{\partial \bar{x}^{m}}{\partial x^{\varepsilon}} \cdots \frac{\partial \bar{x}^{n}}{\partial x^{\nu}} \frac{\partial x^{\alpha}}{\partial \bar{x}^{i}} \frac{\partial x^{\beta}}{\partial \bar{x}^{j}} \cdots \frac{\partial x^{\gamma}}{\partial \bar{x}^{k}} \frac{\partial x^{\omega}}{\partial \bar{x}^{p}}$.

Since the point $q$ is arbitrary, $T_{i j \ldots k, p}^{l m \ldots n}$ is a tensor which is contravariant in $(l, m, \ldots, n)$ and covariant in $(i, j, \ldots, k, p)$.

Let us next evaluate $T_{i j \ldots k, p}^{l m \ldots \ldots}$ in terms of the $\Gamma^{\prime} \mathrm{s}$ and the original tensor $T_{i j \ldots i k}^{l m \ldots}$. To do this we differentiate the equations

$$
t_{i j \ldots k}^{l m \ldots n}=T_{\alpha \beta \ldots \gamma}^{\delta \varepsilon \ldots \nu} \frac{\partial y^{l}}{\partial x^{\delta}} \frac{\partial y^{m}}{\partial x^{\varepsilon}} \cdots \frac{\partial y^{n}}{\partial x^{\nu}} \frac{\partial x^{\alpha}}{\partial y^{i}} \frac{\partial x^{\beta}}{\partial y^{j}} \cdots \frac{\partial x^{\gamma}}{\partial y^{k}},
$$

obtaining

$$
\begin{aligned}
\frac{\partial t_{i j \ldots i}^{l m \ldots}}{\partial y^{p}}= & \frac{\partial T_{\alpha \ldots \nu}^{\delta \ldots \nu}}{\partial x^{\sigma}} \frac{\partial y^{l}}{\partial x^{\delta}} \cdots \frac{\partial y^{n}}{\partial x^{\nu}} \frac{\partial x^{\alpha}}{\partial y^{i}} \cdots \frac{\partial x^{\gamma}}{\partial y^{k}} \frac{\partial x^{\sigma}}{\partial y^{p}} \\
& +T_{\alpha \ldots \nu}^{\delta \ldots \nu} \frac{\partial^{2} y^{l}}{\partial x^{\delta} \partial x^{\sigma}} \frac{\partial x^{\sigma}}{\partial y^{p}} \cdots \frac{\partial y^{n}}{\partial x^{\nu}} \frac{\partial x^{\alpha}}{\partial y^{i}} \cdots \frac{\partial x^{\gamma}}{\partial y^{k}}+\cdots \\
& +T_{\alpha \ldots \nu}^{\delta \ldots \nu} \frac{\partial y^{l}}{\partial x^{\delta}} \cdots \frac{\partial y^{n}}{\partial x^{\nu}} \frac{\partial x^{\alpha}}{\partial y^{i}} \cdots \frac{\partial^{2} x^{\gamma}}{\partial y^{k} \partial y^{p}}
\end{aligned}
$$

At the origin of normal corrdinates

$$
\frac{\partial x^{i}}{\partial y^{j}}=\frac{\partial y^{i}}{\partial x^{j}}=\delta_{j}^{i}, \quad \frac{\partial^{2} y^{i}}{\partial x^{j} \partial x^{k}}=-\frac{\partial^{2} x^{i}}{\partial y^{j} \partial y^{k}}=\left(\Gamma_{j k}^{i}\right)_{0}
$$

as follows directly from (8.5) and its inverse (8.6). The substitution of (10.8) into (10.7) then yields

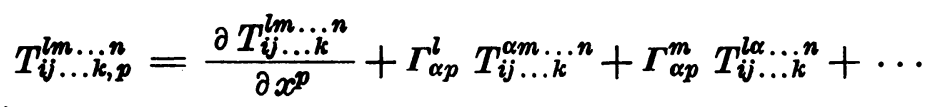

$$
+\Gamma_{\alpha p}^{n} T_{i j \ldots k}^{l m \ldots \alpha}-\Gamma_{i p}^{\alpha} T_{\alpha j \ldots k}^{l m \ldots n}-\Gamma_{j p}^{\alpha} T_{i \alpha \ldots k}^{l m \ldots n}-\ldots-\Gamma_{k p}^{\alpha} T_{i j \ldots \alpha}^{l m \ldots n} .
$$


By using the tensors

$$
D_{\alpha \tau \beta \ldots \nu}^{\sigma a b \ldots n}=\delta_{\alpha}^{\sigma} \delta_{\tau}^{a} \delta_{\beta}^{b} \cdots \delta_{\nu}^{n}+\delta_{\beta}^{\sigma} \delta_{\alpha}^{a} \delta_{\tau}^{b} \cdots \delta_{\nu}^{n}+\cdots+\delta_{\nu}^{\sigma} \delta_{\alpha}^{a} \delta_{\beta}^{b} \cdots \delta_{\tau}^{n}
$$

and

$$
E_{\sigma a b \ldots n}^{\alpha \tau \beta \ldots \nu}=\delta_{\sigma}^{\alpha} \delta_{a}^{\tau} \delta_{b}^{\beta} \ldots \delta_{n}^{\nu}+\delta_{\sigma}^{\beta} \delta_{a}^{\alpha} \delta_{b}^{\tau} \ldots \delta_{n}^{\nu}+\cdots+\delta_{\sigma}^{\nu} \delta_{a}^{\alpha} \delta_{b}^{\beta} \ldots \delta_{n}^{\tau}
$$

the formula (10.9) may be written in the form

$$
\begin{aligned}
& T_{i j \ldots i, p}^{l m \ldots n}=\frac{\partial T_{i j \ldots k}^{l m \ldots n}}{\partial x^{p}}+\Gamma_{\sigma p}^{\tau} T_{i j \ldots k}^{\alpha \beta \ldots \gamma} D_{\alpha \tau \beta \ldots \gamma}^{\sigma l m \ldots n} \\
& -\Gamma_{\tau p}^{\sigma} T_{\alpha \beta \ldots \gamma}^{l m \ldots n} E_{\sigma i j \ldots k}^{\alpha \tau \beta \ldots \gamma}
\end{aligned}
$$

The covariant derivatives of the sum and of the product of two tensors with the same number of covariant and contravariant indices are formed by the same rules as hold in the differential calculus. That is, if

$$
T_{i j \ldots i k}^{l m \ldots n}=A_{i j \ldots i}^{l m \ldots n}+B_{i j \ldots i k}^{l m \ldots n}
$$

then

$$
T_{i j \ldots k, p}^{l m \ldots n}=A_{i j \ldots i, p}^{l m \ldots i n}+B_{i j \ldots i, p}^{l m \ldots n}
$$

and if

$$
T_{i j \ldots k}^{l m \ldots n}=A_{i \ldots s}^{\ell \ldots u} \cdot B_{t \ldots k}^{v \ldots n}
$$

then

$$
T_{i j \ldots k, p}^{l m \ldots n}=A_{i \ldots s}^{l \ldots u} \cdot B_{t \ldots k, p}^{v \ldots n}+A_{i \ldots s, p}^{l \ldots \ldots} \cdot B_{t \ldots k}^{v \ldots n}
$$

These formulas follow without difficulty from (10.1).

11. A generalization of covariant differentiation. By repeated differentiation of (10.3) we obtain

$$
\frac{\partial^{r} \bar{t}_{i j \ldots k}^{l m \ldots n}}{\partial \bar{y}^{p} \ldots \partial \bar{y}^{q}}=\frac{\partial^{r} t_{\alpha \beta \ldots \gamma}^{\delta \varepsilon \ldots \nu}}{\partial y^{\sigma} \ldots \partial y^{\tau}} \frac{\partial \bar{y}^{l}}{\partial y^{\delta}} \frac{\partial \bar{y}^{m}}{\partial y^{\varepsilon}} \ldots
$$

$$
\cdots \frac{\partial \bar{y}^{n}}{\partial y^{\nu}} \frac{\partial y^{\alpha}}{\partial \bar{y}^{i}} \frac{\partial y^{\beta}}{\partial \bar{y}^{j}} \cdots \frac{\partial y^{\gamma}}{\partial \bar{y}^{k}} \frac{\partial y^{\sigma}}{\partial \bar{y}^{p}} \cdots \frac{\partial y^{\tau}}{\partial \bar{y}^{q}}
$$


This shows that a set of tensors $T_{i j \ldots k, \ldots, \ldots}^{l m \ldots n}$ are defined by

$$
T_{i j \ldots k, p \ldots q}^{l m \ldots n}=\left(\frac{\partial^{r} t_{i j \ldots k}^{l m} \ldots n^{n}}{\partial y^{p} \ldots \partial y^{q}}\right)_{0} \quad(r=1,2,3, \ldots)
$$

where the derivatives on the right are evaluated at the origin of normal coordinates. For $r=1, T_{i j \ldots k, \ldots q}^{l m \ldots n}$ is the ordinary covariant derivative that we have just considered. The tensors $T_{i j \ldots k, \ldots \ldots q}^{l m \ldots n}$ form a group of tensors that may be derived from a given tensor. We shall refer to the general tensor of this group, namely, $T_{i j \ldots k, \ldots \ldots q}^{l m \ldots n}$, as the $r$ th extension of $T_{i j \ldots k}^{l m \ldots n}$, $r$ being the number of indices $p, \ldots, q$. By its definition this tensor is symmetric with respect to the indices $p, \ldots, q$. The operation of forming the extension of a tensor may be repeated any number of times. For example, $T_{i j, p q, r, s t u}$ is the third extension of the first extension of the second extension of $T_{i j}$.

The rth extension of the sum of two tensors which are of the same order in their covariant and contravariant indices is equal to the sum of the rth extensions of the two tensors, i. e.,

$$
(A+B)_{i j \ldots k, p \ldots q}^{l m \ldots n}=A_{i j \ldots k, p \ldots q}^{l m \ldots n}+B_{i j \ldots k, \ldots q}^{l m \ldots n}
$$

This follows directly from the character of the tensor transformation. The formula for the covariant derivative (first extension) of the product of two tensors does not apply, however, for the case of the $r$ th extension $(r>1)$. For let the tensor $T$ be equal to the product of two tensors as in the equation (10.15). If $T, A, B$ become $t, a, b$ in a normal coorrdinate system $\left(y^{1}, y^{2}, \ldots, y^{n}\right)$ we have in this system

$$
t_{i j \ldots k}^{l m \ldots n}=a_{i \ldots s}^{l \ldots u} \cdot b_{t \ldots k}^{v \ldots n}
$$

The formula for the $r$ th extension of $T$ is obtained by carrying out the differentiation indicated in the following equation:

$$
\frac{\partial^{r} t_{i j \ldots k}^{l m \ldots n}}{\partial y^{p} \ldots \partial y^{q}}=\frac{\partial^{r}}{\partial y^{p} \ldots \partial y^{q}}\left(a_{i \ldots s}^{l \ldots u} \cdot b_{t \ldots k}^{v \ldots n}\right)
$$

This formula has $2^{r}$ terms.

Any tensor $T_{i j \ldots k, \ldots \ldots q}^{l m \ldots n}$ may be expressed in terms of the $\Gamma^{\prime} \mathrm{s}$ and the original tensor $T_{i j \ldots k}^{l m \ldots \ldots}$ by the same process that we have used for the case 
of the tensor $T_{i j \ldots k, p}^{l m \ldots n}$. That is we have to take the successive partial derivatives of both members of (10.6) and substitute in these equations the equations (10.8) and

$$
\frac{\partial^{3} x^{i}}{\partial y^{j} \partial y^{k} \partial y^{l}}=-\left(\Gamma_{j k l}^{i}\right)_{0}, \quad \frac{\partial^{3} y^{i}}{\partial x^{j} \partial x^{k} \partial x^{l}}=\left(\Delta_{j k l}^{i}\right)_{0},
$$

and so on. It is evident that formulas for extensions of all kinds can be obtained by this process. Instead of giving the general formulas, however, we shall set down the first extensions of the first four kinds for the covariant vector and the covariant tensor of the second order. In these formulas $S$ is used to indicate the sum of all distinct terms which can be formed from the one in the parenthesis by replacing the given combination of the subscripts $p, q$ or $p, q, r$ or $p, q, r, s$ by arbitrary combinations of these subscripts. Thus

$$
S\left(\frac{\partial^{8} T_{i}}{\partial x^{\alpha} \partial x^{p}} \Gamma_{q r}^{\alpha}\right)=\frac{\partial^{2} T_{i}}{\partial x^{\alpha} \partial x^{p}} \Gamma_{q r}^{\alpha}+\frac{\partial^{2} T_{i}}{\partial x^{\alpha} \partial x^{q}} \Gamma_{p r}^{\alpha}+\frac{\partial^{8} T_{i}}{\partial x^{\alpha} \partial x^{r}} \Gamma_{p q}^{\alpha}
$$

$$
T_{i, p}=\frac{\partial T_{i}}{\partial x^{p}}-T_{\alpha} \Gamma_{i p}^{\alpha}
$$

$$
T_{i, v q r}=\frac{\partial^{3} T_{i}}{\partial x^{p}} \frac{\partial x^{q} \partial x^{r}}{}-S\left(\frac{\partial^{2} T_{i}}{\partial x^{\alpha} \partial x^{p}} \Gamma_{q r}^{\alpha}\right)-S\left(\frac{\partial^{2} T_{\alpha}}{\partial x^{p} \partial x^{q}} \Gamma_{i r}^{\alpha}\right)
$$

$$
T_{i, p q}=\frac{\partial^{2} T_{i}}{\partial x^{p} \partial x^{q}}-\frac{\partial T_{i}}{\partial x^{\alpha}} \Gamma_{p q}^{\alpha}-S\left(\frac{\partial T_{\alpha}}{\partial x^{p}} \Gamma_{i q}^{\alpha}\right)-T_{\alpha} \Gamma_{i p q}^{\alpha}
$$

$$
+S\left(\frac{\partial T_{\alpha}}{\partial x^{\beta}} \Gamma_{i p}^{\alpha} \Gamma_{q r}^{\beta}\right)-S\left(\frac{\partial T_{\alpha}}{\partial x^{p}} \Gamma_{i q r}^{\alpha}\right)-\frac{\partial T_{i}}{\partial x^{\alpha}} \Gamma_{p q r}^{\alpha}-T_{\alpha} \Gamma_{i p q r}^{\alpha} ;
$$

$$
T_{i, p q r s}=\frac{\partial^{4} T_{i}}{\partial x^{p} \partial x^{q} \partial x^{r} \partial x^{s}}-S\left(\frac{\partial^{3} T_{i}}{\partial x^{\alpha} \partial x^{p} \partial x^{l}} \Gamma_{r s}^{\alpha}\right)-S\left(\frac{\partial^{3} T_{c}}{\partial x^{p} \partial x^{q} \partial x^{r}} \Gamma_{i s}^{\alpha}\right)
$$

$$
+S\left(\frac{\partial^{2} T_{i}}{\partial x^{\alpha} \partial x^{\beta}} \Gamma_{p q}^{\alpha} \Gamma_{r s}^{\beta}\right)+S\left(\frac{\partial^{2} T_{\alpha}}{\partial x^{\beta} \partial x^{p}} \Gamma_{i q}^{\alpha} \Gamma_{r s}^{\beta}\right)-S\left(\frac{\partial^{2} T_{i}}{\partial x^{\alpha} \partial x^{p}} \Gamma_{q r s}^{\alpha}\right)
$$

$$
\begin{aligned}
& -S\left(\frac{\partial^{2} T_{\alpha}}{\partial x^{p} \partial x^{q}} \Gamma_{i r s}^{\alpha}\right)+S\left(\frac{\partial T_{\alpha}}{\partial x^{\beta}} \Gamma_{i p}^{\alpha} \Gamma_{q r s}^{\beta}\right)+S\left(\frac{\partial T_{\alpha}}{\partial x^{\beta}} \Gamma_{i p q}^{\alpha} \Gamma_{r s}^{\beta}\right) \\
& -S\left(\frac{\partial T_{\alpha}}{\partial x^{p}} \Gamma_{i q r s}^{\alpha}\right)-\frac{\partial T_{i}}{\partial x^{\alpha}} \Gamma_{i p q r s}^{\alpha}-T_{\alpha} \Gamma_{i p q r s}^{\alpha} ;
\end{aligned}
$$


(11.11) $T_{i j, p}=\frac{\partial T_{i j}}{\partial x^{p}}-T_{\alpha j} \Gamma_{i p}^{\alpha}-T_{i \alpha} \Gamma_{j p}^{\alpha}$

$$
T_{i j, p q}=\frac{\partial^{2} T_{i j}}{\partial x^{p} \partial x^{q}}-\frac{\partial T_{i j}}{\partial x^{\alpha}} \Gamma_{p q}^{\alpha}-S\left(\frac{\partial T_{\alpha j}}{\partial x^{p}} \Gamma_{i q}^{\alpha}\right)-S\left(\frac{\partial T_{i \alpha}}{\partial x^{p}} \Gamma_{j q}^{\alpha}\right)
$$

$$
+T_{\alpha \beta} S\left(\Gamma_{i p}^{\alpha} \Gamma_{j q}^{\beta}\right)-T_{\alpha j} \Gamma_{i p q}^{\alpha}-T_{i \alpha} \Gamma_{j p q}^{\alpha}
$$

$$
\begin{aligned}
T_{i j, p q r}= & \frac{\partial^{3} T_{i j}}{\partial x^{p} \partial x^{q} \partial x^{r}}-S\left(\frac{\partial^{2} T_{\alpha j}}{\partial x^{p} \partial x^{q}} \Gamma_{i r}^{\alpha}\right)-S\left(\frac{\partial^{2} T_{i \alpha}}{\partial x^{p} \partial x^{q}} \Gamma_{j r}^{\alpha}\right) \\
& -S\left(\frac{\partial^{2} T_{i j}}{\partial x^{\alpha} \partial x^{p}} \Gamma_{q r}^{\alpha}\right)+\frac{\partial T_{\alpha j}}{\partial x^{\beta}} S\left(\Gamma_{i p}^{\alpha} \Gamma_{q r}^{\beta}\right)+\frac{\partial T_{i \alpha}}{\partial x^{\beta}} S\left(\Gamma_{j p}^{\alpha} \Gamma_{q r}^{\beta}\right)
\end{aligned}
$$

$$
\begin{aligned}
& +S\left(\frac{\partial T_{\alpha \beta}}{\partial x^{p}} \Gamma_{i q}^{\alpha} \Gamma_{j r}^{\beta}\right)-S\left(\frac{\partial T_{\alpha j}}{\partial x^{p}} \Gamma_{i q r}^{\alpha}\right)-S\left(\frac{\partial T_{i \alpha}}{\partial x^{p}} \Gamma_{j q r}^{\alpha}\right) \\
& -\frac{\partial T_{i j}}{\partial x^{\alpha}} \Gamma_{p q r}^{\alpha}+T_{\alpha \beta} S\left(\Gamma_{i p}^{\alpha} \Gamma_{j q r}^{\beta}\right)+T_{\alpha \beta} S\left(\Gamma_{i p q}^{\alpha} \Gamma_{j r}^{\beta}\right) \\
& -T_{\alpha j} \Gamma_{i p q r}^{\alpha}-T_{i \alpha} \Gamma_{j p q r}^{\alpha}
\end{aligned}
$$

$$
T_{i j, p q r s}=\frac{\partial^{4} T_{i j}}{\partial x^{p} \partial x^{q} \partial x^{r} \partial x^{s}}-S\left(\frac{\partial^{3} T_{\alpha j}}{\partial x^{p} \partial x^{q} \partial x^{\gamma}} \Gamma_{i s}^{\alpha}\right)-S\left(\frac{\partial^{s} T_{i \alpha}}{\partial x^{p} \partial x^{q} \partial x^{r}} \Gamma_{j s}^{\alpha}\right)
$$

$$
-S\left(\frac{\partial^{3} T_{i j}}{\partial x^{\alpha} \partial x^{p} \partial x^{q}} \Gamma_{r s}^{\alpha}\right)+S\left(\frac{\partial^{2} T_{\alpha \beta}}{\partial x^{p} \partial x^{q}} \Gamma_{i r}^{\alpha} \Gamma_{j s}^{\beta}\right)+S\left(\frac{\partial^{2} T_{\alpha j}}{\partial x^{\beta} \partial x^{p}} \Gamma_{i q}^{\alpha} \Gamma_{r s}^{\beta}\right)
$$$$
+S\left(\frac{\partial^{2} T_{i \alpha}}{\partial x^{\beta} \partial x^{p}} \Gamma_{j q}^{\alpha} \Gamma_{r s}^{\beta}\right)-S\left(\frac{\partial^{2} T_{i \alpha}}{\partial x^{p} \partial x^{q}} \Gamma_{j r s}^{\alpha}\right)-S\left(\frac{\partial^{2} T_{i j}}{\partial x^{\alpha} \partial x^{p}} \Gamma_{q r s}^{\alpha}\right)
$$$$
+S\left(\frac{\partial^{2} T_{i j}}{\partial x^{\alpha} \partial x^{\beta}} \Gamma_{p q}^{\alpha} \Gamma_{r s}^{\beta}\right)-S\left(\frac{\partial^{2} T_{\alpha j}}{\partial x^{p} \partial x^{q}} \Gamma_{i r s}^{\alpha}\right)-S\left(\frac{\partial T_{\alpha j}}{\partial x^{p}} \Gamma_{i q r s}^{\alpha}\right)
$$

$$
\begin{aligned}
& +S\left(\frac{\partial T_{\alpha \beta}}{\partial x^{p}} \Gamma_{i q r}^{\alpha} \Gamma_{j s}^{\beta}\right)+S\left(\frac{\partial T_{\alpha j}}{\partial x^{\beta}} \Gamma_{i p q}^{\alpha} \Gamma_{r s}^{\beta}\right)+S\left(\frac{\partial T_{\alpha \beta}}{\partial x^{p}} \Gamma_{i q}^{\alpha} \Gamma_{j r s}^{\beta}\right) \\
& -S\left(\frac{\partial T_{\alpha \beta}}{\partial x^{\gamma}} \Gamma_{i p}^{\alpha} \Gamma_{j q}^{\beta} \Gamma_{r s}^{\gamma}\right)+S\left(\frac{\partial T_{\alpha j}}{\partial x^{\beta}} \Gamma_{i p}^{\alpha} \Gamma_{q r s}^{\beta}\right)-S\left(\frac{\partial T_{i \alpha}}{\partial x^{p}} \Gamma_{j q r s}^{\alpha}\right) \\
& +S\left(\frac{\partial T_{i \alpha}}{\partial x^{\beta}} \Gamma_{j p q}^{\alpha} \Gamma_{r s}^{\beta}\right)+S\left(\frac{\partial T_{i \alpha}}{\partial x^{\beta}} \Gamma_{j p}^{\alpha} \Gamma_{q r s}^{\beta}\right)-\frac{\partial T_{i j}}{\partial x^{\alpha}} \Gamma_{p q r s}^{\alpha} \\
& -T_{\alpha j} \Gamma_{i p q r s}^{\alpha}+T_{\alpha \beta} S\left(\Gamma_{i p q r}^{\alpha} \Gamma_{j s}^{\beta}\right)+S\left(T_{\alpha \beta} \Gamma_{i p q}^{\alpha} \Gamma_{j r s}^{\beta}\right) \\
& +T_{\alpha \beta} S\left(\Gamma_{i p}^{\alpha} \Gamma_{j q r s}^{\beta}\right)-T_{i \alpha} \Gamma_{j p q r s}^{\alpha} .
\end{aligned}
$$


12. Formulas for repeated covariant differentiation. In this section we write down a few special formulas relating the tensors obtained by successive covariant differentiation to the higher extensions and the normal tensors. In each case the formula is obtained by computing the covariant derivative in question according to the formulas of $\S 10$ and evaluating at the origin of normal coordinates:

$$
\begin{aligned}
T_{i j, p, q}= & T_{i j, p q}-T_{\alpha j} A_{i p q}^{\alpha}-T_{i \alpha} A_{j p q}^{\alpha} \\
T_{i j, p, q, r}= & T_{i j, p q r}-T_{\alpha j, p} A_{i q r}^{\alpha}-T_{\alpha j, q} A_{i p r}^{\alpha}-T_{\alpha j, r} A_{i p q}^{\alpha} \\
& -T_{i \alpha, p} A_{j q r}^{\alpha}-T_{i \alpha, q} A_{j p r}^{\alpha}-T_{i \alpha, r} A_{j p q}^{\alpha}-T_{i j, \alpha} A_{p q r}^{\alpha} \\
& -T_{\alpha j} A_{i p q r}^{\alpha}-T_{i \alpha} A_{j p q r}^{\alpha} ; \\
T_{i j, p, q, r, s}= & T_{i, p q r s}-T_{\alpha j} A_{i p q r s}^{\alpha}-T_{i \alpha} A_{j p q r s}^{\alpha} \\
& -T_{\alpha j, q} A_{i p r s}^{\alpha}-T_{\alpha j, r} A_{i p q s}^{\alpha}-T_{\alpha j, s} A_{i p q r}^{\alpha} \\
& -T_{i \alpha, q} A_{j p r s}^{\alpha}-T_{i \alpha, r} A_{j p q s}^{\alpha}-T_{i \alpha, s} A_{j p q r}^{\alpha}
\end{aligned}
$$




$$
\begin{aligned}
& -T_{\alpha j, p} A_{i q r s}^{\alpha}-T_{i \alpha, p} A_{j q r s}^{\alpha}-T_{i j, \alpha} A_{p q r s}^{\alpha} \\
& -T_{r i, q r} A_{i p s}^{\alpha}-T_{r i, q s} A_{i p r}^{\alpha}-T_{c i, r s} A_{i p q}^{\alpha} \\
& -T_{i \kappa, q r} A_{j p s}^{\alpha}-T_{i \kappa, q s} A_{j p r}^{\alpha}-T_{i \kappa, r s} A_{j p q}^{\alpha} \\
& -T_{\alpha j, p, q} A_{i r s}^{\alpha}-T_{\alpha j, p, r} A_{i q s}^{\alpha}-T_{\alpha j, p, s} A_{i q r}^{\alpha} \\
& -T_{i \kappa, p, q} A_{j r s}^{\alpha}-T_{i \kappa, p, r} A_{j q s}^{\alpha}-T_{i \alpha, p, s} A_{j q r}^{\alpha} \\
& -T_{i j, \alpha, q} A_{p r s}^{\alpha}-T_{i j, \alpha, r} A_{p q s}^{\alpha}-T_{i j, \alpha, s} A_{p q r}^{\alpha} \\
& -T_{i j, p, \omega} \mathrm{A}_{g r s}^{\prime \prime}
\end{aligned}
$$

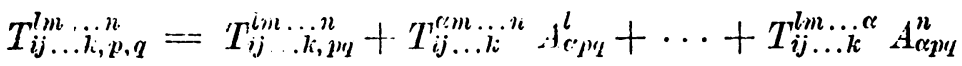

$$
\begin{aligned}
& -T_{\alpha j \ldots k}^{l m \ldots n} A_{i p q}^{\alpha}+\cdots+T_{i j \ldots \alpha}^{l m \ldots n} A_{k p q}^{\alpha} .
\end{aligned}
$$

13. A generalization of the normal tensors. If we transform the equations (6.3) to a system of normal coördinates and make use of (7.1) we obtain the following sequence:

$$
\begin{array}{r}
C_{\alpha \beta}^{i} \xi^{\alpha} \xi^{\beta}=0, \\
C_{\alpha \beta \gamma}^{i} \xi^{\alpha} \xi^{\beta} \xi^{\gamma}=0,
\end{array}
$$

$$
C_{\alpha \beta \gamma \ldots \sigma}^{i} \xi^{\alpha} \xi^{\beta} \xi^{\gamma} \ldots \xi^{\sigma}=0,
$$

where the $C$ 's denote the corresponding functions $\Gamma$ in normal coordinates. $C_{\alpha \beta \gamma}^{i} \ldots \sigma$ is symmetric in the indices $\alpha \beta \gamma \ldots \sigma$. The functions $C_{\alpha \beta}^{i}$ are related 
to the $\Gamma$ 's by (7.5) and there are similar equations of transformation for the other $C$ 's. Thus

$$
\begin{aligned}
& C_{\alpha \beta \gamma}^{\nu} \frac{\partial x^{i}}{\partial y^{\nu}}=\frac{\partial^{\beta} x^{i}}{\partial y^{\alpha} \partial y^{\beta} \partial y^{\gamma}}+I_{\mu \nu \tau}^{i} \frac{\partial x^{\mu}}{\partial y^{\alpha}} \frac{\partial x^{\nu}}{\partial y^{\beta}} \frac{\partial x^{\tau}}{\partial y^{\gamma}} \\
&-\frac{\partial^{2} x^{i}}{\partial y^{\mu} \partial y^{\alpha}} C_{\beta \gamma}^{\mu}-\frac{\partial^{2} x^{i}}{\partial y^{\mu} \partial y^{\beta}} C_{\gamma^{\alpha}}^{\mu}-\frac{\partial^{2} x^{i}}{\partial y^{\mu} \partial y^{\gamma}} C_{\alpha \beta}^{\mu} .
\end{aligned}
$$

Since the $\xi$ 's are entirely arbitrary at the origin of normal coordinates it follows from (13.1) that

$$
\left(C_{\alpha \beta \gamma \ldots \sigma}^{i}\right)_{0}=0
$$

where the left member denotes the value of $C$ at the origin of the normal coordinate system.

We may define a set of functions $\left.A_{(\alpha \beta \gamma}^{i} \ldots \sigma\right) p \ldots q$ of $\left(x^{1}, x^{2}, \ldots, x^{n}\right)$ corresponding to the normal tensor $A_{\alpha \beta p \ldots q}^{i}$ defined by (9.3) by the equations

$$
A_{(\alpha \beta \gamma \ldots \sigma) p \ldots q}^{i}=\left(\frac{\partial^{n} C_{\alpha \beta \gamma \ldots \sigma}^{i}}{\partial y^{p} \ldots \partial y^{q}}\right)_{0}
$$

in which the derivative on the right is evaluated at the origin of normal coorrdinates. By a method similar to that employed in $\S 9$ we can show that $A_{(\alpha \beta \gamma \ldots \sigma) p \ldots q}^{i}$ possesses a tensor character, but this fact may also be inferred by observing that $A_{(\alpha \beta \gamma \ldots \sigma) p \ldots q}^{i}$ is expressible in terms of the normal tensors. The tensors $A_{(\alpha \beta \gamma \ldots \sigma) p \ldots q}^{i}$ thus constitute a generalization of the normal tensors. They are symmetric in the indices $\alpha \beta \gamma \ldots \sigma$ and $p \cdots q$. In case $A_{(\alpha \beta \gamma \ldots \sigma) p \ldots q}^{i}$ contains only two terms in the parenthesis it is a normal tensor and we shall then omit the parenthesis for simplicity.

The following equations express a few particular cases of the relations between the tensors $A_{(\alpha \beta \gamma \ldots \sigma) p \ldots q}^{i}$ and the normal tensors. These equations are obtained by differentiating the identities (6.5) referred to normal coördinates and evaluating at the origin. The symbols $P$ and $S$ have their previous significance (cf. $\S 6$ and $\S 11$ ) except that $P$ operates only on the letters $\alpha \beta \gamma \delta \varepsilon$ and $S$ only on the letters $p, q, r$ :

$$
A_{(\alpha \beta \gamma) p}^{i}=\frac{1}{3} P\left(A_{\alpha \beta \gamma p}^{i}\right)
$$


$A_{(\alpha \beta \gamma) p q}^{i}=\frac{1}{3} P\left[A_{\alpha \beta \gamma p q}^{i}-2 S\left(A_{\nu \alpha p}^{i} A_{\beta \gamma q}^{\nu}\right)\right]$ $A_{(\alpha \beta \gamma) p q r}^{i}=\frac{1}{3} P\left[A_{\alpha \beta \gamma p q r}^{i}-2 S\left(A_{\nu \alpha p q}^{i} A_{\beta \gamma r}^{\nu}\right)-2 S\left(A_{\nu \alpha p}^{i} A_{\beta \gamma q r}^{\nu}\right)\right]$ $A_{(\alpha \beta \gamma \delta) p}^{i}=\frac{1}{4} P\left(A_{(\alpha \beta \gamma) \delta p}^{i}\right)$

$$
A_{(\alpha \beta \gamma \delta) p q}^{i}=\frac{1}{4} P\left[A_{(\alpha \beta \gamma) \delta p q}^{i}-3 S\left(A_{(\nu \alpha \beta) p}^{i} A_{\gamma \delta q}^{\nu}\right)\right]
$$

(13.10) $A_{(\alpha \beta \gamma \delta \varepsilon) p}^{i}=\frac{1}{5} P\left(A_{\alpha \beta \gamma \delta \varepsilon p}^{i}\right)$

By differentiating the equations of the type (13.2) and evaluating at the origin of normal coördinates we may express these tensors in terms of the functions $\boldsymbol{r}$. For example

$$
\begin{gathered}
A_{(\alpha \beta \gamma) p}^{i}=-\Gamma_{\alpha \beta \gamma p}^{i}+\frac{\partial \Gamma_{\alpha \beta \gamma}^{i}}{\partial x^{p}}-\Gamma_{\mu \alpha \beta}^{i} \Gamma_{\gamma p}^{\mu}-\Gamma_{\mu \alpha \gamma}^{i} \Gamma_{\beta p}^{\mu}-\Gamma_{\mu \beta \gamma}^{i} \Gamma_{\alpha p}^{\mu} \\
+\Gamma_{\mu \alpha}^{i} A_{\beta \gamma p}^{\mu}+\Gamma_{\mu \beta}^{i} A_{\gamma \alpha p}^{\mu}+\Gamma_{\mu \gamma}^{i} A_{\alpha \beta p}^{\mu} .
\end{gathered}
$$

The generalized normal tensors appear in some of the formulas of extension which generalize the formulas of $\S 12$. We here write down only the following four particular cases:

$$
\begin{aligned}
& T_{i, p, q r}=T_{i, p q r}-T_{\alpha, q} A_{i p r}^{\alpha}-T_{\alpha, r} A_{i p q}^{\alpha}-T_{\alpha} A_{i p q r}^{\alpha} \\
& T_{i, p q, r}=T_{i, p q r}-T_{i, \alpha} A_{p q r}^{\alpha}-T_{\alpha, p} A_{i q r}^{\alpha}-T_{\alpha, q} A_{i p r}^{\alpha}-T_{\alpha} A_{(i p q) r}^{\alpha} \\
& T_{i j, p, q r}=T_{i, p q r}-T_{\alpha j, q} A_{i p r}^{\alpha}-T_{\alpha j, r} A_{i p q}^{\alpha}-T_{i \alpha, q} A_{j p r}^{\alpha}-T_{i \alpha, r} A_{j p q}^{\alpha} \\
& -T_{\alpha j} A_{i p q r}^{\alpha}-T_{i \alpha} A_{j p q r}^{\alpha} \\
& T_{i, p q, r}=T_{i j, p q r}-T_{i j, \alpha} A_{p q r}^{\alpha}-T_{\alpha j, p} A_{i q r}^{\alpha}-T_{\alpha j, q} A_{i p r}^{\alpha}-T_{i \alpha, p} A_{j q r}^{\alpha} \\
& -T_{i \alpha, q} A_{j p r}^{\alpha}-T_{\alpha j} A_{(i p q) r}^{\alpha}-T_{i \alpha} A_{(j p q) r}^{\alpha} .
\end{aligned}
$$


The generalized normal tensors satisfy the identity

$$
S\left(A_{(\alpha \beta \gamma \ldots \sigma) p \ldots q}^{i}\right)=0
$$

where $S(\quad)$ denotes the sum of the terms obtainable from the one in the parenthesis which are not identical because of the symmetric properties of $A_{(\alpha \beta \gamma \ldots \sigma) p \ldots q}^{i}$. This identity may easily be proved by the method used for the corresponding theorem about the normal tensors in $\S 9$.

14. The curvature tensor. The normal tensor $A_{j k l}^{i}$ is related to the curvature tensor by the equation

$$
B_{j k l}^{i}=A_{j k l}^{i}-A_{j l k}^{i}
$$

which is immediately evident on comparing (2.7) with (9.13). The tensor character of $B$ follows from that of $A$. From the definition it follows that

$$
B_{j k l}^{i}=-B_{j l k}^{i}
$$

From (9.9) it follows that

$$
B_{j k l}^{i}+B_{k l j}^{i}+B_{l j k}^{i}=0
$$

Also by solving the equations (14.1) and (9.9) for the $A$ 's we obtain

$$
A_{j k l}^{i}=\frac{1}{3}\left(2 B_{j k l}^{i}+B_{l j k}^{i}\right)
$$

or

$$
A_{j k l}^{i}=\frac{1}{3}\left(B_{j k l}^{i}+B_{k j l}^{i}\right)
$$

If we write (9.13) in normal coördinates, differentiate, and evaluate at the origin we obtain

$$
A_{j k l, m}^{i}=A_{j k l m}^{i}-A_{(j k l) m}^{i}
$$

From this and (14.1) it follows that

$$
B_{j k l, m}^{i}=A_{j k l m}^{i}-A_{j l k m}^{i}
$$


The equations (14.7) and (9.10) may be solved for the $A$ 's giving

$$
A_{j k l m}^{i}=\frac{\dot{1}}{6}\left(5 B_{k j l, m}^{i}+4 B_{l k j, m}^{i}+3 B_{l j m, k}^{i}+2 B_{m l k, j}^{i}+B_{m k j, l}^{i}\right) .
$$

If in (14.7) we permute the indices $k, l, m$ cyclically and add the three resulting equations we obtain the identity of Bianchi,

$$
B_{j k l, m}^{\alpha}+B_{j l m, k}^{\alpha}+B_{j m k, l}^{\alpha}=0
$$

From (14.7) there also follows the identity,

$$
B_{i j k, l}^{\alpha}+B_{k i l, j}^{\alpha}+B_{l k j, i}^{\alpha}+B_{j l i, k}^{\alpha}=0
$$

From (12.7) there follows the important identity of Ricci and Levi-Civita,

$$
\begin{aligned}
& T_{i j \ldots k, p, q}^{l m \ldots n}-T_{i j \ldots k, q, p}^{l m \ldots \ldots n}=T_{i j \ldots k}^{\alpha m \ldots n} B_{\alpha p q}^{l}+\cdots+T_{i j \ldots k}^{l m \ldots \alpha} B_{\alpha p q}^{n} \\
& -T_{\alpha j \ldots k}^{l m \ldots n} B_{i p q}^{\alpha}-\ldots-T_{i j \ldots \alpha}^{l m \ldots n} B_{k p q}^{\alpha} \text {. }
\end{aligned}
$$

Using the tensors $D$ and $E(14.11)$ becomes

$T_{i j \ldots k, p, q}^{l m \ldots n}-T_{i j \ldots k, q, p}^{l m \ldots n}=T_{i j \ldots k}^{\alpha \beta \ldots \gamma} B_{\sigma p q}^{\tau} D_{\alpha \tau \beta \ldots \gamma}^{\sigma l m \ldots n}-T_{\alpha \beta \ldots \gamma}^{l m \ldots n} B_{\tau p q}^{\sigma} E_{\sigma i j \ldots k}^{\alpha \tau \beta \ldots \gamma}$

This identity may be generalized by combining identities of the type(13.12). For example,

$$
T_{i, p, q r}-T_{i, q, p r}=T_{\alpha, p} A_{i q r}^{\alpha}-T_{\alpha, q} A_{i p r}^{\alpha}-T_{\alpha, r} B_{i p q}^{\alpha}-T_{\alpha} B_{i p q, r}^{\alpha} .
$$

15. Homogeneous first integrals. A homogeneous first integral of the $k$ th degree of the differential equations (2.1) is an equation of the form

$$
a_{\alpha \beta \ldots \gamma} \frac{d x^{\alpha}}{d s} \frac{d x^{\beta}}{d s} \ldots \frac{d x^{\gamma}}{d s}=\text { constant }
$$

which holds along every path. From the equations for the transformation of $d x^{i} / d s$ it follows that the functions $a_{\alpha \beta \ldots \gamma}$ are the components of a covariant tensor. We shall now derive some general theorems about the conditions 
under which a tensor $a_{\alpha \beta \ldots \gamma}$ gives rise to a first integral. The first of these theorems is given by Ricci and Levi-Civita for the case of the Riemann geometry in Chapter 5 of their Méthodes de calcul differentiel absolu.

If we differentiate (15.1) with respect to $s$ we have

$$
\frac{d a_{\alpha \beta \ldots \gamma}}{d s} \frac{d x^{\alpha}}{d s} \frac{d x^{\beta}}{d s} \ldots \frac{d x^{\gamma}}{d s}+a_{\alpha \beta \ldots \gamma} \frac{d^{2} x^{\alpha}}{d s^{2}} \frac{d x^{\beta}}{d s} \ldots \frac{d x^{\gamma}}{d s}+\ldots=0 .
$$

At the origin of the normal coördinates this equation becomes

$$
\frac{\partial a_{\alpha \beta \ldots \gamma}}{\partial y^{\sigma}} \xi^{\alpha} \xi^{\beta} \ldots \xi^{\gamma} \xi^{\sigma}=0
$$

owing to the equation (7.1). We may also write

$$
a_{\alpha \beta \ldots \gamma, \sigma} \xi^{\alpha} \xi^{\beta} \ldots \xi^{\gamma} \xi^{\sigma}=0
$$

where $a_{\alpha \beta \ldots \gamma, \sigma}$ is the covariant derivative of $a_{\alpha \beta \ldots \gamma}$. The substitution involved in obtaining the last equation is permissible on account of (10.1) which holds at the origin of the normal coordinates. The identity

$$
P\left(a_{\alpha \beta \ldots \gamma, \sigma}\right)=0
$$

where $P$ indicates the sum of the terms obtained from the one inside the parenthesis by cyclic permutation of the subscripts, is therefore a necessary condition for the existence of the integral (15.1). Owing to its tensor character (15.3) has validity in all coordinate systems.

To show that (15.3) is also sufficient for the existence of the first integral, let this equation be satisfied by the symmetric tensor $a_{i j . . . k}$, and express it in its expanded form

$$
P\left(\frac{\partial a_{i j \ldots k}}{\partial x^{l}}-\Gamma_{i l}^{\alpha} a_{\alpha j \ldots k}-\Gamma_{j l}^{\alpha} a_{i \alpha \ldots k}-\ldots-\Gamma_{l l}^{\alpha} a_{i j \ldots \alpha}\right)=0 .
$$

If we consider this equation referred to a normal coordinate system and multiply by $\left(d y^{i} / d s\right)\left(d y^{j / d s}\right) \cdots\left(d y^{k} / d s\right)\left(d y^{l} / d s\right)$, we obtain

$$
\frac{d a_{\alpha \beta \ldots \gamma}}{d s} \frac{d y^{\alpha}}{d s} \frac{d y^{\beta}}{d s} \cdots \frac{d y^{\gamma}}{d s}=0
$$


on making use of the equation (7.6). Since the derivatives $d y^{i} / d s$ in (15.4) are constant along any particular path, it follows that

$$
a_{\alpha \beta \ldots \gamma} \frac{d y^{\alpha}}{d s} \frac{d y^{\beta}}{d s} \ldots \frac{d y^{\gamma}}{d s}=\text { constant }
$$

along any particular path. In consequence of the tensor character of $a_{i j \ldots k}$, we have in general coorrdinates

$$
a_{\alpha \beta \ldots \gamma} \frac{d x^{\alpha}}{d s} \frac{d x^{\beta}}{d \varepsilon} \ldots \frac{d x^{\gamma}}{d s}=\text { constant. }
$$

Hence, $A$ necessary and sufficient condition for the existence of a homogeneous first integral of the kth degree is that a symmetric covariant tensor of the kth order $a_{i j . . . k}$ exist which satisfies (15.3).

If a symmetric tensor $b_{i j \ldots k}$ and function $\varphi\left(x^{1}, x^{2}, \ldots, x^{n}\right)$ exist which satisfy the equations

$$
P\left(b_{i j \ldots k, l}\right)=P\left(b_{i j \ldots k} \varphi_{l}\right), \quad \varphi_{l}=\frac{\partial \varphi}{\partial x^{l}},
$$

where $b_{i j \ldots k, l}$ is the covariant derivative of $b_{i j \ldots k}$, a function $\psi$ can be chosen so that the equation

$$
P\left[\left(\psi b_{i j \ldots k}\right)_{l}\right]=0
$$

is satisfied. The bracket contains the covariant derivative of $\psi b_{i j \ldots k}$ with respect to $x^{l}$. In fact, we have

$$
\begin{aligned}
& P\left[\left(\psi b_{i j \ldots k}\right)_{l}\right] \\
& \quad=P\left(\psi_{l} b_{i j \ldots k}+\psi b_{i j \ldots k, l}\right) \\
& \quad=\psi P\left[b_{i j \ldots k}\left(\frac{\partial \log \psi}{\partial x^{l}}+\frac{\partial \varphi}{\partial x^{l}}\right)\right] .
\end{aligned}
$$

Hence (15.6) is satisfied if we put

$$
\psi=e^{-\varphi}
$$


Therefore if (15.5) is satisfied a first integral exists which is given by $e^{-\varphi} b_{i j \ldots k}$. That (15.5) is a necessary condition is immediate, for, as we have seen, if $b_{i j} . . k$ furnishes a first integral (15.5) is satisfied with $\varphi=$ constant.

Hence, $A$ necessary and sufficient condition for the existence of a covariant tensor $a_{i j . . . k}$ which satisfies (15.3) is that a covariant tensor $b_{i j . . . k}$ and function $\varphi$ exist which satisfy (15.5). If the tensor $b_{i j} \ldots k$ and function $\varphi$ exist, then

$$
a_{i j \ldots k}=e^{-\varphi} b_{i j \ldots k} .
$$

A particular case of (15.3) is

$$
a_{i j \ldots k, l}=0
$$

where $a_{i j \ldots k, l}$ is the covariant derivative of $a_{i j \ldots k}$. In a manner similar to the above it can then be shown that

$$
b_{i j \ldots k, l}=b_{i j \ldots k} \varphi_{l}, \quad \varphi_{l}=\frac{\partial \varphi}{\partial x^{l}}
$$

is a necessary and sufficient condition for the existence of a first integral which satisfies (15.7), and that this integral is given by $a_{i j \ldots k}=e^{-\varphi} b_{i j . . k}$.

Hence, $A$ necessary and sufficient condition for the existence of a covariant tensor $a_{i j . . . k}$ which satisfies (15.7) is that a covariant tensor $b_{i j . . . k}$ and function $\varphi$ exist which satisfy (15.8). If the tensor $b_{i j . . . k}$ and function $\varphi$ exist then

$$
a_{i j \ldots k}=e^{-\varphi} b_{i j \ldots k} .
$$

The equation (14.12) provides a new statement of this last theorem. If the tensor $b_{i j . . . k}$ satisfies (15.8) we obtain by covariant differentiation

$$
\begin{aligned}
b_{i j \ldots k, l, m} & =b_{i j \ldots k, m} \varphi_{l}+b_{i j \ldots k} \varphi_{l, m} \\
& =b_{i j \ldots k}\left(\varphi_{l} \varphi_{m}+\varphi_{l, m}\right) .
\end{aligned}
$$

Hence,

$$
b_{i j \ldots k, l, m}-b_{i j \ldots k, m, l}=0
$$

From (14.12) we then have

$$
b_{\alpha \beta \ldots \gamma} B_{\tau l m}^{\sigma} E_{\sigma i j \ldots k}^{\alpha \tau \beta \ldots \gamma}=0
$$


Conversely, if the tensor $b_{i j \ldots k}$ and vector $\varphi_{l}$ satisfy (15.9) and

$$
b_{i j \ldots k, l}=b_{i j \ldots k} \varphi_{l},
$$

where $b_{i j \ldots k, l}$ is the covariant derivative of $b_{i j . . . k}$, we have

$$
b_{i j \ldots k, l, m}=b_{i j \ldots k}\left(\varphi_{l} \varphi_{m}+\varphi_{l, m}\right)
$$

and

$$
b_{i j \ldots k, l, m}-b_{i j \ldots k, m, l}=b_{i j \ldots k}\left(\varphi_{l, m}-\varphi_{m, l}\right)
$$

Since $b_{i j . . . k}$ satisfies (15.9)

$$
b_{i j \ldots k, l, m}-b_{i j \ldots k, m, l}=0
$$

so that

$$
\varphi_{l, m}-\varphi_{m, l}=0
$$

or

$$
\frac{\partial \varphi_{l}}{\partial x^{m}}=\frac{\partial \varphi_{m}}{\partial x^{l}}
$$

and this last equation is the condition that $\varphi_{l}$ be the gradient of a scalar function $\varphi\left(x^{1}, x^{2}, \ldots, x^{n}\right)$, i. e.,

$$
\Upsilon_{l}=\frac{\partial \varphi}{\partial x^{l}}
$$

Hence, $A$ necessary and sufficient condition for the existence of a covariant tensor $a_{i j . . . k}$ which satisfies (15.7) is that a covariant tensor $b_{i j . . . k}$ and vector $\varphi_{l}$ exist which satisfy (15.9) and (15.10). If the tensor $b_{i j \ldots k}$ and vector $\varphi_{l}$ exist, then

$$
a_{i j \ldots k}=e^{-\varphi} b_{i j \ldots k}
$$

16. Algebraic condition for existence of first integrals of a particular class. We shall now derive a condition on the functions $r$ for the existence of a homogeneous first integral of the $k$ th degree which satisfies the particular condition (15.7). The condition is to involve only the algebraic consistency of a set of tensor equations formed from the functions $\Gamma$. If the 
covariant tensor of the $k$ th order $a_{i j . . k}$ satisfies the condition (15.7) it follows by (14.12) that $a_{i j . . k}$ will satisfy a sequence of equations of the form

$$
\begin{aligned}
a_{\alpha \beta \ldots \gamma} D_{i j \ldots k l m}^{\alpha \beta \ldots \gamma} & =0, \\
a_{\alpha \beta \ldots \gamma} D_{i j \ldots k m, r_{1}}^{\alpha \beta \ldots \gamma} & =0, \\
a_{\alpha \beta \ldots \gamma} D_{i j \ldots k l m, r_{1}, r_{2}}^{\alpha \beta \ldots \gamma} & =0,
\end{aligned}
$$

$$
a_{\alpha \beta \ldots \gamma} D_{i j \ldots k i m, r_{1}, r_{2} \ldots, r_{n}}^{\alpha \beta \ldots \gamma}=0
$$

where

$$
D_{i j \ldots i m}^{\alpha \beta \ldots \gamma}=B_{\tau l m}^{\sigma} E_{\sigma i j \ldots k}^{\alpha \tau \beta \ldots \gamma}
$$

and $D_{i j \ldots k l m, r_{1}, r_{2}, \ldots, r_{n}}^{\alpha \beta \ldots \gamma r e s e n t s ~ t h e ~} n$th covariant derivative of $D_{i j \ldots k l m}^{\alpha \beta \ldots \gamma}$. The algebraic consistency of the equations (16.1) is a necessary condition on the $\Gamma$ 's for the existence of the homogeneous first integral of the $k$ th degree which satisfies (15.7).

The algebraic solutions of the equations (16.1) possess a tensor character. For let $a_{i j . . . k}$ represent an algebraic solution of (16.1). Under a general transformation of coordinates the first set of equations of (16.1) becomes

$$
\bar{a}_{\alpha \beta \ldots \gamma} \bar{D}_{i j \ldots k l m}^{\alpha \beta \ldots \gamma}=0
$$

where $\bar{D}_{i j \ldots k l m}^{\alpha \beta \ldots \gamma}$ is defined by the equations of transformation

$$
D_{p q \ldots r s t}^{i j \ldots k}=\frac{\partial x^{i}}{\partial \bar{x}^{\alpha}} \cdots \frac{\partial x^{k}}{\partial \bar{x}^{\beta}} \frac{\partial \bar{x}^{\gamma}}{\partial x^{p}} \cdots \frac{\partial \bar{x}^{\delta}}{\partial x^{t}} \bar{D}_{\gamma \ldots \delta}^{\alpha \ldots \beta}
$$


and $\bar{a}_{\alpha \beta \ldots \gamma}$ represents an algebraic solution of (16.2). Substituting (16.3) in the first set of equations of (16.1) we obtain

$$
a_{\varepsilon \ldots \omega} \frac{\partial x^{\varepsilon}}{\partial \bar{x}^{\mu}} \cdots \frac{\partial x^{\omega}}{\partial \bar{x}^{\nu}} \frac{\partial \bar{x}^{\gamma}}{\partial x^{p}} \cdots \frac{\partial \bar{x}^{\delta}}{\partial x^{t}} \bar{D}_{\gamma \ldots \nu}^{\mu \ldots \nu}=0
$$

If we multiply (16.4) by $\left(\partial x^{p / \partial \bar{x}^{i}}\right)\left(\partial x^{q / \partial \bar{x}^{j}}\right) \cdots\left(\partial x^{t} / \partial \bar{x}^{m}\right)$ and sum for $(p, q, \ldots, t)$, then

$$
a_{\varepsilon \ldots \omega} \frac{\partial x^{\varepsilon}}{\partial \bar{x}^{\mu}} \ldots \frac{\partial x^{\omega}}{\partial \bar{x}^{\nu}} \bar{D}_{i j \ldots k l m}^{\mu \ldots \nu}=0
$$

and a comparison of these equations with (16.2) shows that a solution of (16.2) is given by

$$
\bar{a}_{i j \ldots k}=a_{\varepsilon \ldots \omega} \frac{\partial x^{\varepsilon}}{\partial \bar{x}^{i}} \cdots \frac{\partial x^{\omega}}{\partial \bar{x}^{k}}
$$

While we have considered the first set of equations of (16.1) a similar result would have been obtained with regard to any other set. Hence the algebraic solutions of (16.1) are tensors and it is consequently permissible to form the covariant derivative of these solutions as we shall do in the later work.

Let us now assume the algebraic consistency of the equations (16.1) and suppose that the first system of these equations admits a set of fundamental solutions denoted by $b_{i j \ldots k}^{(p)}, p=1,2, \ldots, s$. The general solution of this system of equations can then be expressed as a linear combination of the fundamental solutions $b_{i j \ldots k}^{(p)}$ with arbitrary functional coefficients. We next consider the first and second systems of equations (16.1) and suppose that these equations have a fundamental set of solutions $c_{i j \ldots k}^{(p)}, p=1,2, \ldots, t$, in which of course $s \geqq t$. If $s=t$ then $c_{i j \ldots k}^{(p)}, p=1,2, \ldots, t$ will furnish a fundamental set of solutions of the first system of equations which satisfies the second system. If $s>t$ we consider the first three systems of equations, which we may suppose to have a fundamental set of solutions $d_{i j}^{(p)} \ldots k, p=1$, $2, \ldots, u$, with the condition $t \geqq u$. In case $t=u$ then $d_{i j \ldots k}^{(p)}, p=1,2$, $\ldots, u$, will furnish a fundamental set of solutions of the first two systems of equations which satisfies the third system. By proceeding in this way we shall finally come to a point where the first $N$ systems of equations of (16.1) will admit a fundamental set of solutions which satisfies the system immediately following in the sequence. Hence to say that the equations (16.1) are 
algebraically consistent implies that there is a number $N$ such that the first $N$ systems of equations (16.1) admit a fundamental set of solutions $a_{i j \ldots k}^{(p)}, p=1$, $2, \ldots, s$, which satisfies the equation

$$
a_{\alpha \beta \ldots \gamma} D_{i j \ldots k l m, r_{1}, r_{\mathbf{s}}, \ldots, r_{N}}^{\alpha \beta \ldots \gamma}=0
$$

The general solution of the first $N$ systems of equations is then

$$
a_{i j \ldots k}=\psi^{(\alpha)} a_{i j \ldots k}^{(\alpha)} \quad(\alpha=1,2, \ldots, s),
$$

where the expression on the right is summed for $\alpha$, and $\varphi^{(\alpha)}$ is an arbitrary function of $\left(x^{1}, x^{2}, \ldots, x^{n}\right)$.

Before proceeding further with the general case let us consider the particular case where the first system of equations (16.1) has a unique solution $a_{i j \ldots k}$ which satisfies the second system, i. e.,

$$
a_{\alpha \beta \ldots \gamma} D_{i j \ldots k l m, r_{1}}^{\alpha \beta \ldots \gamma}=0
$$

Under these conditions a homogeneous first integral of the $k$ th degree will exist whose covariant derivative vanishes. For if we differentiate the first system of equations (16.1) covariantly we obtain, on account of (16.6),

$$
a_{\alpha \beta \ldots \gamma, r} D_{i j . . k l m}^{\alpha \beta \ldots \gamma}=0
$$

where $a_{\alpha \beta \ldots \gamma, \gamma}$ is the covariant derivative of $a_{\alpha \beta \ldots \gamma}$. Since (16.7) possesses a unique solution $a_{i j \ldots k}$, it follows that

$$
a_{i j \ldots k, l}=\varphi_{l} a_{i j \ldots k}
$$

in which $\varphi_{l}$ is a covariant vector. The above statement then follows from the last theorem of $\S 15$.

Going back to the general case let us substitute one of the fundamental solutions $a_{i j}^{(p)}$...k in the equations of the sequence (16.1) through the $(N+1)$ th. We may then differentiate these equations covariantly so as to obtain the following: 


$$
a_{\alpha \beta \ldots \gamma, r}^{(p)} D_{i j \ldots k l m}^{\alpha \beta \ldots \gamma}=0
$$$$
a_{\alpha \beta \ldots \gamma, r}^{(p)} D_{i j \ldots k l m, r_{1}}^{\alpha \beta \ldots \gamma}=0
$$

$$
a_{\alpha \beta \ldots \gamma, r}^{(p)} D_{i j \ldots k l m, r_{1}, r_{2}, \ldots, r_{N-1}}^{\alpha \beta \ldots \gamma}=0
$$

where $a_{\alpha \beta \ldots, \gamma, r}^{(p)}$ is the covariant derivative of $a_{\alpha \beta \ldots \gamma}^{(p)}$. Since $a_{i j \ldots k, l}^{(p)}$ is a solution of (16.8) it may be expressed linearly in terms of the fundamental solutions of these equations. Hence

$$
a_{i j \ldots k, l}^{(p)}=\lambda l^{(p \alpha)} a_{i j \ldots k}^{(\alpha)}
$$

where the expression on the right is summed for $\alpha$, and the $\lambda$ 's are covariant vectors. Since $a_{i j . . . k}^{(p)}$ satisfies the first system of the sequence (16.1),

$$
a_{\hat{v}, \ldots k, l, m}^{(p)}-a_{v, \ldots k, m, l}^{(p)}=0
$$

If $a_{y, \ldots k, l}^{(p)}$ as given by (16.9) be differentiated covariantly and substituted in (16.10) there is obtained the following condition on the $\lambda$ 's:

$$
\frac{\partial \lambda_{k}^{(p q)}}{\partial x^{l}}-\frac{\partial \lambda_{l}^{(p q)}}{\partial x^{k}}+\lambda_{k}^{(p \alpha)} \lambda_{l}^{(\alpha q)}-\lambda_{l}^{(p \alpha)} \lambda_{k}^{(\alpha q)}=0
$$

If we substitute (16.5) in (15.7) we see that it will be satisfied if a set of functions $\varphi^{(p)}, p=1,2, \ldots, s$ can be chosen so as to satisfy the equations

$$
\frac{\partial \varphi^{(p)}}{\partial x^{k}}+\varphi^{(\alpha)} \lambda_{k}^{(\alpha p)}=0
$$

Such a set of functions can be chosen, for in consequence of (16.11) these equations are completely integrable. This set of functions $\varphi^{(\alpha)}$ will determine 
according to (16.5) a covariant tensor of the $k$ th degree $a_{i j . . k}$ whose covariant derivative vanishes.

Hence, a necessary and sufficient condition for the existence of a homogeneous first integral of the kth degree $a_{i j . . . k}$ which satisfies (15.7) is that there exists a number $N$ such that the first $N$ systems of equations (16.1) admit a fundamental set of $s$ solutions $(s \geqq 1)$ which satisfy the $(N+1)$ th system of equations.

17. Special cases. The theorems of the last two sections have some interesting applications in the linear and quadratic cases. It is natural to define a field of parallel covariant vectors by means of a set of functions $h_{i}$ such that

$$
h_{i, j}=0 \text {. }
$$

For this means that if normal coordinates are introduced with origin at an arbitrary point, we have at this point

$$
\frac{d h_{i}}{d s}=\frac{\partial h_{i}}{\partial y^{\alpha}} \frac{d y^{\alpha}}{d s}=0
$$

By the third theorem in italics in $\S 15$, a necessary and sufficient condition for the existence of a field of parallel covariant vectors is the existence of a function $\varphi$ and vector $A_{i}$ such that*

$$
A_{i, j}=A_{i} \varphi_{j}, \quad \varphi_{j}=\frac{\partial \varphi}{\partial x^{j}}
$$

The last theorem of $\S 15$ now shows that a necessary and sufficient condition for a field of parallel covariant vectors $h_{i}$ is that a covariant vector $A_{i}$ exist which satisfies the equations

$$
\begin{aligned}
& A_{i, j}=A_{i} \varphi_{j}, \\
& A_{\alpha} B_{i j k}^{\alpha}=0,
\end{aligned}
$$

where $\varphi_{j}$ is a covariant vector and $A_{i, j}$ is the covariant derivative of $A_{i}$. The theorem of $\S 16$ shows that a necessary and sufficient condition for a field of

* Eisenhart, Proceedings of the National Academy of Sciences, vol. 8 (1922), pp. 207-212, defines $A_{1}$ as a field of parallel vectors, and finds the condition (17.5) for their existence. 
parallel covariant vectors is that there exists a number $N$ such that the first $N$ sets of equations of the sequence

$$
\begin{aligned}
A_{\alpha} B_{i j k}^{\alpha} & =0, \\
A_{\alpha} B_{i j k, l}^{\alpha} & =0,
\end{aligned}
$$

$$
A_{\alpha} B_{i j k, l, m}^{\alpha}=0 \text {, }
$$

admit a fundamental set of $s$ solutions $(s \geqq 1)$ which satisfy the $(N+1)$ th set. In particular a sufficient condition is obtained if the first system of equations of (17.6) be algebraically consistent and all their solutions satisfy the second system of these equations.

Going now to the quadratic case we see from the third theorem in italics in $\S 15$ that the condition on the functions $\Gamma$ for the geometry of paths to become a Riemann geometry is that a tensor $g_{i j}$ exist such that

$$
g_{i j, k}=g_{i j} \varphi_{k}, \quad \boldsymbol{\varphi}_{k}=\frac{\partial \varphi}{\partial x^{k}} .
$$

The equation (17.7) without the condition that the vector $\rho_{k}$ be the gradient of a scalar function gives the geometry upon which Weyl bases his electremagnetic and gravitational theory, for this equation is equivalent to the equation (2.10). By the last theorem of $\S 15$, the condition (17.7) can be written

$$
\begin{gathered}
g_{i j, k}=g_{i j} \varphi_{k}, \\
g_{\alpha j} B_{i k l}^{\alpha}+g_{i \alpha} B_{j k l}^{\alpha}=0 .
\end{gathered}
$$

This shows furthermore that a necessary and sufficient condition for the Weyl geometry to become the Riemann geometry is that the tensor $g_{i j}$ satisfy (17.9).

The theorem of $\S 16$ shows that a necessary and sufficient condition for the geometry of paths to become a Riemann geometry is that there exists a number $N$ such that the first $N$ systems of equations of the following sequence 
admit a fundamental set of $s$ solutions $(s \geqq 1)$ which satisfy the $(N+1)$ st system of equations:

$$
\begin{aligned}
g_{\alpha j} B_{i k l}^{\alpha}+g_{i \alpha} B_{j k l}^{\alpha} & =0 \\
g_{\alpha j} B_{i k l, m}^{\alpha}+g_{i \alpha} B_{j k l, m}^{\alpha} & =0 \\
g_{\alpha j} B_{i k l, m, n}^{\alpha}+g_{i \alpha} B_{j k l, m, n}^{\alpha} & =0
\end{aligned}
$$

In particular* we have that a sufficient condition for the geometry of paths to become a Riemann geometry is that the equations

$$
g_{\alpha j} B_{i k l}^{\alpha}+g_{i \alpha} B_{j k l}^{\alpha}=0
$$

be algebraically consistent and that all their solutions satisfy

$$
g_{\alpha j} B_{i k l, m}^{\alpha}+g_{i \alpha} B_{j k l, m}^{\alpha}=0
$$

18. The homogeneous linear first integral. From the first theorem of $\S 15$ it follows that a necessary and sufficient condition for the covariant vector $h_{i}$ to furnish a linear first integral,

$$
h_{\alpha} \frac{d x^{\alpha}}{d s}=\text { constant }
$$

is that the equation

$$
h_{i . j}+h_{j, i}=0
$$

be satisfied, i. e., the covariant derivative $h_{i, j}$ must be skew symmetric in the indices $i$ and $j$. The equations (14.12) show that

$$
h_{i, j, k}-h_{i, k, j}=h_{\alpha} B_{i k j}^{\alpha}
$$

* Eisenhart and Veblen, Proceedings of the National Academy of Sciences, vol. 8 (1922), pp. 19-23. 
By (18.2) these give rise to

$$
\begin{aligned}
& h_{i, j, k}+h_{k, i, j}=h_{\alpha} B_{i k j}^{\alpha}, \\
& h_{k, i, j}+h_{j, k, i}=h_{\alpha} B_{k j i}^{\alpha}, \\
& h_{j, k, i}+h_{i, j, k}=h_{\alpha} B_{j i k}^{\alpha} .
\end{aligned}
$$

If we add these three equations we obtain

$$
h_{i, j, k}+h_{j, k, i}+h_{k, i, j}=0 \text {. }
$$

Combining (18.5) with the second equation of (18.4), we have also

$$
h_{i, j, k}=h_{\alpha} B_{k i j}^{\alpha}
$$

These are integrability conditions obtained by consideration of second derivatives. In order to obtain those involving third derivatives we use (13.12), which with (18.2) gives

$$
\begin{aligned}
& h_{i, p q r}+h_{p, i q r}=2 h_{\alpha, q} A_{i p r}^{\alpha}+2 h_{\alpha, r} A_{i p q}^{\alpha}+2 h_{\alpha} A_{i p q r}^{\alpha} \\
& h_{q, i p r}+h_{i, q p r}=2 h_{\alpha, p} A_{q i r}^{\alpha}+2 h_{\alpha, r} A_{q i p}^{\alpha}+2 h_{\alpha} A_{q i p r}^{\alpha}, \\
& h_{p, q i r}+h_{q, p i r}=2 h_{\alpha, i} A_{p q r}^{\alpha}+2 h_{\alpha, r} A_{p q i}^{\alpha}+2 h_{\alpha} A_{p q i r}^{\alpha} .
\end{aligned}
$$

If we add the first two of these equations and subtract the third, we obtain

$$
h_{i, p q r}=h_{\alpha, p} A_{i q r}^{\alpha}+h_{\alpha, q} A_{i p r}^{\alpha}-h_{\alpha, i} A_{p q r}^{\alpha}
$$

$$
+h_{\alpha, r}\left(A_{i p q}^{\alpha}+A_{q i p}^{\alpha}-A_{p q i}^{\alpha}\right)+h_{\alpha}\left(A_{i p q r}^{\alpha}+A_{q i p r}^{\alpha}-A_{p q i r}^{\alpha}\right) .
$$


Now interchange the indices $q$ and $r$ and subtract the resulting equation from this one. We obtain$$
h_{\alpha, i} B_{p r q}^{\alpha}+h_{\alpha, p} B_{i q r}^{\alpha}+h_{\alpha, q} B_{r p i}^{\alpha}+h_{\alpha, r} B_{q i p}^{\alpha}+h_{\alpha}\left(B_{q i p, r}^{\alpha}+B_{r p i, q}^{\alpha}\right)=0
$$

If we collect the terms in the equation (18.9) we have

$$
h_{\alpha} C^{\alpha}+h_{\alpha, \beta} D^{\alpha \beta}=0
$$

where

$$
\begin{aligned}
& C^{\alpha}=B_{\gamma \delta \varepsilon, \mu}^{\alpha}\left(\delta_{k}^{\gamma} \delta_{i}^{\delta} \delta_{j}^{\varepsilon} \delta_{l}^{\mu}+\delta_{l}^{\gamma} \delta_{j}^{\delta} \delta_{i}^{\varepsilon} \delta_{k}^{\mu}\right), \\
& D^{\alpha \beta}=B_{\gamma \delta \varepsilon}^{\alpha}\left(\delta_{i}^{\beta} \delta_{j}^{\gamma} \delta_{l}^{\delta} \delta_{k}^{\varepsilon}+\delta_{j}^{\beta} \delta_{i}^{\gamma} \delta_{k}^{\delta} \delta_{l}^{\varepsilon}+\delta_{k}^{\beta} \delta_{l}^{\gamma} \delta_{j}^{\delta} \delta_{i}^{\varepsilon}+\delta_{l}^{\beta} \delta_{k}^{\gamma} \delta_{i}^{\delta} \delta_{j}^{\varepsilon}\right) .
\end{aligned}
$$

$C^{\alpha}$ is a tensor which is contravariant of the first order and covariant of the fourth, $D^{\alpha \beta}$ is a tensor which is contravariant of the second order and covariant of the fourth. The covariant indices of these tensors have been omitted for simplicity. If we differentiate (18.10) covariantly, we obtain

$$
h_{\alpha i} C^{\alpha}+h_{\alpha} C_{, i}^{\alpha}+h_{\alpha, \beta, i} D^{\alpha \beta}+h_{\alpha, \beta} D_{, i}^{\alpha \beta}=0
$$

and this becomes

$$
h_{\alpha, i} C^{\alpha}+h_{\alpha} C_{, i}^{\alpha}+h_{\gamma} B_{i \alpha \beta}^{\gamma} D^{\alpha \beta}+h_{\alpha, \beta} D_{, i}^{\alpha \beta}=0
$$

when we make the substitution (18.6). This equation may be written in an abbreviated form as follows:

$$
h_{\alpha} C_{1}^{\alpha}+h_{\alpha, \beta} D_{1}^{\alpha \beta}=0
$$

Covariant differentiation of (18.12) will give rise to a new equation which can in its turn be abbreviated to the form (18.10), this process requiring the use of (18.6) to eliminate $h_{\alpha, \beta, \gamma}$. Continuing in this way we obtain an infinite sequence of equations. For the purpose of convenient reference we shall write this sequence with the equation (18.2) as the first member: 


$$
\begin{aligned}
& h_{\alpha, \beta}+h_{\beta, \alpha}=0, \\
& h_{\alpha} C^{\alpha}+h_{\alpha, \beta} D^{\alpha \beta}=0 \text {, } \\
& h_{\alpha} C_{1}^{\alpha}+h_{\alpha, \beta} D_{1}^{\alpha \beta}=0 \text {, } \\
& h_{\alpha} C_{n}^{\alpha}+h_{\alpha, \beta} D_{n}^{\alpha \beta}=0 \text {, }
\end{aligned}
$$

The algebraic consistency of this set of equations, regarded as equations for the determination of $h_{i}$ and $h_{i, j}$, is a necessary condition for the existence of a first integral $h_{i}$. Hence as in $\S 16$ there must be a value of $N$ such that the first $N+1$ sets of equations admit a fundamental set of solutions $h_{i}^{(p)}, h_{i j}^{(p)}(p=1,2, \ldots, s)$ each of which will satisfy the system of equations next following in the sequence (18.13). This necessary condition turns out also to be a sufficient condition.

Before proving this in general, let us consider the special case in which $N=1$ and $s=1$. In this case (18.2) and (18.10) are consistent and possess a unique algebraic solution consisting of a set of functions $h_{i}$, $i=1,2, \ldots, n$, and a set of functions $h_{i j}, i, j=1,2, \ldots, n$, which satisfy (18.11). It may now be shown that the solutions $h_{i}$ and $h_{i j}$ are tensors, so that it is possible to substitute these quantities in the equation (18.10) and differentiate it covariantly. Doing this we obtain

$$
h_{\alpha, i} C^{\alpha}+h_{\alpha} C_{, i}^{\alpha}+h_{\alpha \beta, i} D^{\alpha \beta}+h_{\alpha \beta} D_{, i}^{\alpha \beta}=\mathbf{0} .
$$

If we subtract (18.14) from (18.11) with $h_{i j}$ replacing $h_{i, j}$, we obtain

$$
\left(h_{\alpha i}-h_{\alpha, i}\right) C^{\alpha}+\left(h_{\gamma} B_{i \alpha \beta}^{\gamma}-h_{\alpha \beta, i}\right) D^{\alpha \beta}=0 .
$$


In (18.15) the coefficient of $D^{\alpha \beta}$ is skew symmetric in the indices $\alpha$ and $\beta$. By hypothesis, the solution of (18.15) and (18.2) is unique and consequently the solution $\left(h_{\alpha i}-h_{\alpha, i}\right),\left(h_{\gamma} B_{i \alpha \beta}^{\gamma}-h_{\alpha \beta, i}\right)$ can only differ from the solution $h_{\alpha}, h_{\alpha \beta}$ by a factor of multiplication. Hence

$$
\begin{gathered}
h_{i j}-h_{i, j}=\varphi_{j} h_{i}, \\
h_{\alpha} B_{k i j}^{\alpha}-h_{i, j, k}=\varphi_{k} h_{i j},
\end{gathered}
$$

where $\varphi_{i}$ is a covariant vector. If we differentiate (18.16) covariantly, obtaining

$$
h_{i j, k}-h_{i, j, k}=\varphi_{j, k} h_{i}+\varphi_{j} h_{i, k}
$$

and from this form the expression

$$
\left(\varphi_{j, k}-\varphi_{k, j}\right) h_{i}=h_{i j, k}-h_{i k, j}+h_{i, k, j}-h_{i, j, k}+\varphi_{k} h_{i, j}-\varphi_{j} h_{i, k}
$$

we find on substituting the equations (18.16) and (18.17) in the right member of this equation that it vanishes identically. The functions $h_{i}$ are not all identically zero, for if so it would follow by (18.16) that the functions $h_{i j}$ are also identically zero, contrary to the assumption that (18.2) and (18.10) are algebraically consistent. Hence

$$
\varphi_{j, k}-\varphi_{k, j}=0
$$

The vector $\varphi_{i}$ is therefore the gradient of a scalar function $\varphi$, i.e.,

$$
\varphi_{i}=\frac{\partial \varphi}{\partial x^{i}}
$$

Now we shall have a first integral if a function $\psi$ exists such that

$$
\left(\psi h_{i}\right)_{j}=\psi h_{i j}
$$


where $\left(\psi h_{i}\right)_{j}$ denotes the covariant derivative of $\psi h_{i}$. For if this equation is satisfied, $\psi h_{i}$ will be a covariant vector satisfying (18.2) and hence will give a first integral. Expanding (18.18)

$$
\frac{\partial \psi}{\partial x^{j}} h_{i}+\psi h_{i, j}=\psi h_{i j}
$$

or

$$
h_{i j}-h_{i, j}=\Psi_{j} h_{i}
$$

where

$$
\boldsymbol{\psi}_{j}=\frac{\partial \psi \boldsymbol{\psi}}{\partial x^{j}} \text { and } \Psi=\log \psi
$$

The gradient $\Psi_{j}$ is a covariant vector and consequently (18.19) will be satisfied if we put

$$
\Psi=\varphi .
$$

Hence, a sufficient condition for the existence of a linear first integral is that (18.2) and (18.10) be algebraically consistent and that they possess a unique solution which satisfies (18.11).

Let us now return to the general case and assume that there is a value of $N$ such that the first $N+1$ systems of equations (18.13) admit a fundamental system of solutions $h_{i}^{(p)}, h_{i}^{(p)}, p=1,2, \ldots, s$, each of which satisfies the system of equations immediately following in the sequence. By the same argument as before, $h_{i}^{(p)}$ and $h_{i j}^{(p)}$ are tensors for all values of $p$. The general solution of the first $N+1$ systems of equations is then

$$
h_{i}=\varphi^{(\alpha)} h_{i}^{(\alpha)}
$$

$$
h_{i j}=\varphi^{(\alpha)} h_{i j}^{(\alpha)},
$$

where the terms on the right are summed for $\alpha$ from $\alpha=1$ to $\alpha=s$. If we differentiate the equation

$$
h_{\alpha}^{(p)} C^{\alpha}+h_{\alpha \beta}^{(p)} D^{\alpha \beta}=0
$$


covariantly, and subtract it from the equation

$$
h_{\alpha}^{(p)} C_{1}^{\alpha}+h_{\alpha \beta}^{(p)} D_{1}^{\alpha \beta}=0
$$

we obtain

$$
\left(h_{\alpha i}^{(p)}-h_{\alpha, i}^{(p)}\right) C^{\alpha}+\left(h_{\gamma}^{(p)} B_{i \alpha \beta}^{\gamma}-h_{\alpha \beta, i}^{(p)}\right) D^{\alpha \beta}=0
$$

If we next differentiate (18.23) covariantly and subtract it from the equation immediately following in the sequence, we have

$$
\left(h_{\alpha i}^{(p)}-h_{\alpha, i}^{(p)}\right) C^{\alpha}+\left(h_{\gamma}^{(p)} B_{i \alpha \beta}^{\gamma}-h_{\alpha \beta, i}^{(p)}\right) D^{\alpha \beta}=0
$$

Continuing in this way we obtain the equations

$$
\begin{aligned}
& \left(h_{\alpha i}^{(p)}-h_{\alpha, i}^{(p)}\right) C^{\alpha}+\left(h_{\gamma}^{(p)} B_{i \alpha \beta}^{\gamma}-h_{\alpha \beta, i}^{(p)}\right) D^{\alpha \beta}=0, \\
& \left(h_{\alpha i}^{(p)}-h_{\alpha, i}^{(p)}\right) C_{1}^{\alpha}+\left(h_{\gamma}^{(p)} B_{i \alpha \beta}^{\gamma}-h_{\alpha \beta, i}^{(p)}\right) D_{1}^{\alpha \beta}=0, \\
& \left(h_{\alpha i}^{(p)}-h_{\alpha, i}^{(p)}\right) C_{n}^{\alpha}+\left(h_{\gamma}^{(p)} B_{i \alpha \beta}^{\gamma}-h_{\alpha \beta, i}^{(p)}\right) D_{n}^{\alpha \beta}=0,
\end{aligned}
$$

The term $\left(h_{\gamma}^{(p)} B_{i \alpha \beta}^{\gamma}-h_{\alpha \beta, i}^{(p)}\right)$ is skew symmetric in the indices $\alpha$ and $\beta$, and we may therefore express the quantities $\left(h_{\alpha i}^{(p)}-h_{\alpha, i}^{(p)}\right),\left(h_{\gamma}^{(p)} B_{i \alpha \beta}^{\gamma}-h_{\alpha \beta, i}^{(p)}\right)$ as a linear combination of the particular solutions $h_{\alpha}^{(p)}, h_{\alpha \beta}^{(p)}, p=1,2, \ldots, s$ :

$$
\begin{aligned}
h_{i, j}^{(p)}-h_{i j}^{(p)} & =\lambda_{j}^{(p \alpha)} h_{i}^{(\alpha)}, \\
h_{i, k}^{(p)}-h_{\alpha}^{(p)} B_{k i j}^{\alpha} & =\lambda_{k}^{(p \alpha)} h_{i j}^{(\alpha)} .
\end{aligned}
$$


To determine the condition which the covariant vectors $\lambda_{k}^{(i j)}$ must satisfy we differentiate (18.25) covariantly, ubtaining

$$
h_{i, p, q}^{(k)}=h_{i p, q}^{(k)}+\lambda_{p, q}^{(k \alpha)} h_{i}^{(\alpha)}+\lambda_{p}^{(k \alpha)} h_{i, q}^{(\alpha)}
$$

or

$(18.27) h_{i, p, q}^{(k)}=h_{\alpha}^{(k)} B_{q i p}^{\alpha}+\lambda_{q}^{(k \alpha)} h_{i p}^{(\alpha)}+\lambda_{p, q}^{(k \alpha)} h_{i}^{(\alpha)}+\lambda_{p}^{(k \alpha)}\left(h_{i q}^{(\alpha)}+\lambda_{q}^{(\alpha \beta)} h_{i}^{(\beta)}\right)$.

If we interchange $p$ and $q$ in (18.27) and subtract these two equations we find that

$$
h_{i}^{(\alpha)}\left(\lambda_{p, q}^{(k \alpha \alpha)}-\lambda_{q, p}^{(k \alpha)}+\lambda_{p}^{(k \beta)} \lambda_{q}^{(\beta \alpha)}-\lambda_{q}^{(k \beta)} \lambda_{p}^{(\beta \alpha)}\right)=0
$$

We next differentiate (18.26) covariantly,

$$
h_{i p, q, r}^{(k)}=h_{\alpha, r}^{(k)} B_{q i p}^{\alpha}+h_{\alpha}^{(k)} B_{q i p, r}^{\alpha}+\lambda_{q, r}^{(k \alpha)} h_{i p}^{(\alpha)}+\lambda_{q}^{(k \alpha)} h_{i p, r}^{(\alpha)}
$$

or

$$
\begin{aligned}
h_{i p, q, r}^{(k)}=\left(h_{\alpha \gamma}^{(k)}+\lambda_{r}^{(k \beta)} h_{\alpha}^{(\beta)}\right) B_{q i p}^{\alpha}+h_{\alpha}^{(k)} & B_{q i p, r}^{\alpha}+\lambda_{q, r}^{(k \alpha)} h_{i p}^{(\alpha)} \\
& +\lambda_{q}^{(k \alpha)}\left(h_{\beta}^{(\alpha)} B_{r i p}^{\beta}+\lambda_{r}^{(\alpha \beta)} h_{i p}^{(\beta)}\right) .
\end{aligned}
$$

Interchanging $r$ and $q$ in (18.29) and subtracting the two equations,

$$
h_{i p}^{(\alpha)}\left(\lambda_{q, r}^{(k \alpha)}-\lambda_{r, q}^{(k \alpha)}+\lambda_{q}^{(k \beta)} \lambda_{r}^{(\beta \alpha)}-\lambda_{r}^{(k \beta)} \lambda_{q}^{(\beta \alpha)}\right)
$$

$$
+h_{\alpha}^{(k)}\left(B_{q i p, r}^{\alpha}-B_{r i p, q}^{\alpha}\right)+h_{\alpha r}^{(k)} B_{q i p}^{\alpha}+h_{\alpha q}^{(k)} B_{r p i}^{\alpha}+h_{\alpha p}^{(k)} B_{i q r}^{\alpha}+h_{\alpha i}^{(k)} B_{p r q}^{\alpha}=0 .
$$

This equation reduces to

$$
h_{i p}^{(\alpha)}\left(\lambda_{q, r}^{(k \alpha)}-\lambda_{r, q}^{(k \alpha)}+\lambda_{q}^{(k \beta)} \lambda_{r}^{(\beta \alpha)}-\lambda_{r}^{(k \beta)} \lambda_{q}^{(\beta \alpha)}\right)=0
$$

since $h_{i}^{(\alpha)}, h_{i j}^{(\alpha)}$ is a solution of (18.9). From (18.28) and (18.31) we now deduce that

$$
\lambda_{q, r}^{(k \alpha)}-\lambda_{r, q}^{(k \alpha)}+\lambda_{q}^{(k \beta)} \lambda_{r}^{(\beta \alpha)}-\lambda_{r}^{(k \beta)} \lambda_{q}^{(\beta \alpha)}=0
$$


for if (18.32) were not satisfied there would be a linear relation among the solutions $h_{i}^{(k)}, h_{i p}^{(k)}$, contrary to the hypothesis that $h_{i}^{(k)}, h_{i p}^{(k)}, k=1,2, \ldots, s$, is a fundamental set of solutions.

A linear first integral $h_{i}$ will be determined by (18.20) if $\varphi^{(\alpha)}$ can be chosen so that the equations

$$
\left(\varphi^{(\alpha)} h_{i}^{(\alpha)}\right)_{p}=\varphi^{(\alpha)} h_{i p}^{(\alpha)}
$$

are satisfied, where the term on the left is the covariant derivative of $\varphi^{(\alpha)} h_{i}^{(\alpha)}$, for the covariant vector $h_{i}=\varphi^{(\alpha)} h_{i}^{(\alpha)}$ will possess a covariant derivative $h_{i, p}$ which is skew symmetric in $i$ and $p$. Expanding (18.33)

$$
\frac{\partial \varphi^{(\alpha)}}{\partial x^{p}} h_{i}^{(\alpha)}+\varphi^{(\alpha)}\left(h_{i, p}^{(\alpha)}-h_{i p}^{(\alpha)}\right)=0
$$

From (18.25) we find that the condition on the $\varphi$ 's can be put in the form

$$
\frac{\partial \varphi^{(\beta)}}{\partial x^{p}}+\varphi^{(\alpha)} \lambda_{p}^{(\alpha \beta)}=0
$$

The integrability conditions of (18.35) are the equations (18.32) and hence a set of $\varphi$ 's can be found which will satisfy (18.33).

Hence, a necessary and sufficient condition for the existence of a linear first integral (18.1) is that the $\Gamma$ 's be such that there exists a number $N$ such that the first $N+1$ systems of equations (18.13) admit a fundamental set of $s$ solutions $(s \geqq 1)$ which satisfies the $(N+2)$ nd system of equations.

19. The homogeneous quadratic first integral. A necessary and sufficient condition for the existence of a homogeneous quadratic first integral

$$
g_{\alpha \beta} \frac{d x^{\alpha}}{d s} \frac{d x^{\beta}}{d s}=\text { constant }
$$

such that

$$
g_{i j}=g_{j i}
$$

is that $g_{i j}$ satisfy the condition

$$
g_{i j, p}+g_{j p, i}+g_{p i, j}=0,
$$


where $g_{i j, p}$ is the covariant derivative of $g_{i j}$. By differentiating (19.3) covariantly, we obtain

$$
\begin{array}{r}
g_{i j, p, q}+g_{j p, i, q}+g_{p i, j, q}=0, \\
g_{i j, p, q, r}+g_{j p, i, q, r}+g_{p i, j, q, r}=0, \\
g_{i j, p, q, r, s}+g_{j p, i, q, r, s}+g_{p i, j, q, r, s}=0 .
\end{array}
$$

By substituting (12.4), (12.5) and (12.6) in these three equations we obtain

where

$$
\begin{aligned}
P_{i j p q}= & 2\left(g_{i \alpha} A_{j p q}^{\alpha}+g_{j \alpha} A_{p i q}^{\alpha}+g_{p \alpha} A_{i j q}^{\alpha}\right) \\
P_{i j p q r}= & 2\left(g_{i \alpha, q} A_{j p r}^{\alpha}+g_{j \alpha, q} A_{p i r}^{\alpha}+g_{p \alpha, q} A_{i j r}^{\alpha}\right. \\
& +g_{i \alpha, r} A_{j p q}^{\alpha}+g_{j \alpha, r} A_{p i q}^{\alpha}+g_{p \alpha, r} A_{i j q}^{\alpha} \\
& \left.+g_{i \alpha} A_{j p q r}^{\alpha}+g_{j \alpha} A_{p i q r}^{\alpha}+g_{p \alpha} A_{i j q r}^{\alpha}\right) \\
P_{i j p q r s} & =2\left(g_{i \alpha, q r} A_{j p s}^{\alpha}+g_{i \alpha, r s} A_{j p q}^{\alpha}+g_{i \alpha, s q} A_{j p r}^{\alpha}\right. \\
& +g_{j \alpha, q r} A_{i p s}^{\alpha}+g_{j \alpha, r s} A_{i p q}^{\alpha}+g_{j \alpha, s q} A_{i p r}^{\alpha} \\
& +g_{p \alpha, q r} A_{i j s}^{\alpha}+g_{p \alpha, r s} A_{i j q}^{\alpha}+g_{p \alpha, s q} A_{i j r}^{\alpha} \\
& +g_{i \alpha, q} A_{j p r s}^{\alpha}+g_{i \alpha, r} A_{j p q s}^{\alpha}+g_{i \alpha, s} A_{j p q r}^{\alpha} \\
& +g_{j \alpha, q} A_{i p r s}^{\alpha}+g_{j \alpha, r} A_{i p q s}^{\alpha}+g_{j \alpha, s} A_{i p q r}^{\alpha} \\
& +g_{p \alpha, q} A_{i j r s}^{\alpha}+g_{p \alpha, r} A_{i j q s}^{\alpha}+g_{p \alpha, s} A_{i j q r} \\
& \left.+g_{i \alpha} A_{j p q r s}^{\alpha}+g_{j \alpha} A_{i p q r s}^{\alpha}+g_{p \alpha} A_{i j q r s}^{\alpha}\right)
\end{aligned}
$$


The $P$ 's are tensors which are symmetric in the first three indices and also in the remaining ones. Thus

$$
\begin{gathered}
P_{i j p q}=P_{j i p q}=P_{i p j q}, \\
P_{i j p q r}=P_{j i p q r}=P_{i j p r q} \text { etc., } \\
P_{i j p q r s}=P_{j i p q r s}=P_{i j p q g r} \text { etc. }
\end{gathered}
$$

The equations (19.4) can not be solved for $g_{i j, p q}$ but may be solved for the difference of two of these extensions, namely

$$
g_{i j, p q}-g_{p q, i j}=\frac{1}{2}\left(P_{i j p q}+P_{i j q p}-P_{j p q i}-P_{i p q j}\right) .
$$

We may however solve* (19.5) for $g_{i j, p q r}$, thus

$$
\begin{aligned}
g_{i j, p q r}=\frac{1}{3} & \left(P_{i j p q r}+P_{i j q p r}+P_{i j r p q}+P_{p q r i j}\right) \\
& -\frac{1}{6}\left(P_{i p q j r}+P_{i r p j q}+P_{i q r j p}+P_{j p q i r}+P_{j r p i q}+P_{j q r i p}\right) .
\end{aligned}
$$

Similarly from (19.6)

$$
\begin{aligned}
g_{i j, p q r s}= & \frac{1}{3}\left(P_{i j p q r s}+P_{i j q p r s}+P_{i j r p q s}+P_{p q r i j s}\right) \\
& -\frac{1}{6}\left(P_{i p q j r s}+P_{i r p j q s}+P_{i q r j p s}+P_{j p q i r s}+P_{j r p i q s}+P_{j q r i p s}\right) .
\end{aligned}
$$

The equations (19.11) and (19.12) coustitute integrability conditions arising from second and third derivatives respectively. By interchanging $r$ and $s$ in (19.13) and subtracting we obtain the integrability conditions aristog from the fourth derivatives:

$$
\begin{gathered}
\left(2 P_{i j r q p s}+2 P_{p q r i j s}+P_{i s p j q r}+P_{i q s i p r}+P_{j s p i q r}+P_{j q s i p r}\right) \\
-\left(2 P_{i j g q p r}+2 P_{p q s i j r}+P_{i r p j q s}+P_{i q r j p s}+P_{j r p i q s}+P_{j q r i p s}\right)=0 .
\end{gathered}
$$

* The solution is facilitated by noticing that the tensors in the left member of (19.5) can be regarded as notation for the vertices of a Desargues configuration. 
If we substitute the value of $P_{i j p q r s}$ given by (19.9) in (19.14) we obtain an equation which we may write in the form

$$
g_{\alpha \beta} U_{i j p q r s}^{\alpha \beta}+g_{\alpha \beta, \gamma} \nabla_{i j p q r s}^{\alpha \beta \gamma}+g_{\alpha \beta, \gamma \delta} W_{i j p q r s}^{\alpha \beta \gamma \delta}=0
$$

where $U, V$, and $W$ are tensors.

Let us next consider the identity

$$
g_{i j, p, q}=g_{i j, p q}-g_{\alpha j} A_{i p q}^{\alpha}-g_{i \alpha} A_{j p q}^{\alpha}
$$

(cf. (12.4)). The third covariant derivative $g_{i j, p, q, r}$ may be evaluated in terms of $g_{i j}$ and $g_{i j, p}$ by setting the value of $g_{i j, p q r}$ given by (19.12) in the identity (12.5):

$$
\begin{aligned}
g_{i j, p, q, r}= & g_{\alpha i, p} A_{j r q}^{\alpha}+g_{\alpha i, q} A_{j r p}^{\alpha}+g_{\alpha i, r} A_{j q p}^{\alpha}+g_{\alpha j, p} A_{r i q}^{\alpha}+g_{\alpha j, q} A_{r i p}^{\alpha} \\
& +g_{\alpha j, r} A_{q i p}^{\alpha}+g_{\alpha p, i} A_{q r j}^{\alpha}+g_{\alpha p, j} A_{q r i}^{\alpha}+g_{\alpha p, q} A_{i j r}^{\alpha}+g_{\alpha p, r} A_{i j q}^{\alpha} \\
& +g_{\alpha q, i} A_{r p j}^{\alpha}+g_{\alpha q, j} A_{r p i}^{\alpha}+g_{\alpha q, p} A_{i j r}^{\alpha}+g_{\alpha q, r} A_{i j p}^{\alpha}+g_{\alpha r, i} A_{p q j}^{\alpha} \\
& +g_{\alpha r, j} A_{p q i}^{\alpha}+g_{\alpha r, p} A_{i j q}^{\alpha}+g_{\alpha r, q} A_{i j p}^{\alpha}-g_{i j, \alpha} A_{p q r}^{\alpha} \\
& +g_{\alpha i}\left(A_{j q p r}^{\alpha}+A_{j r p q}^{\alpha}\right)+g_{\alpha j}\left(A_{q i p r}^{\alpha}+A_{r i q p}^{\alpha}\right) \\
& +g_{\alpha p}\left(A_{i j q r}^{\alpha}+A_{q r i j}^{\alpha}\right)+g_{\alpha q}\left(A_{i j p r}^{\alpha}+A_{p r i j}^{\alpha}\right) \\
& +g_{\alpha r}\left(A_{i j p q}^{\alpha}+A_{p q i j}^{\alpha}\right) .
\end{aligned}
$$

If we differentiate both members of (19.16) covariantly and substitute for $g_{i j, p, q, r}$ its value from (19.17), we obtain an equation which we may write as

$$
g_{i j, p q, r}=g_{\alpha \beta} E_{i j p q r}^{\alpha \beta}+g_{\alpha \beta, \gamma} F_{i j p q r}^{\alpha \beta \gamma}
$$

where $E$ and $F$ are tensors. Next differentiate (19.15) covariantly, obtaining

$$
g_{\alpha \beta, i} U^{\alpha \beta}+g_{\alpha \beta} U_{, i}^{\alpha \beta}+g_{\alpha \beta, \gamma, i} V^{\alpha \beta \gamma}+g_{\alpha \beta, \gamma} \nabla_{, i}^{\alpha \beta \gamma}
$$

$$
+g_{\alpha \beta, \gamma \sigma, i} W^{\alpha \beta \gamma \sigma}+g_{\alpha \beta, \gamma \sigma} W_{, i}^{\alpha \beta \gamma \sigma}=0
$$


in which we have omitted the covariant indices for simplicity. When we make the substitutions (19.16) and (19.18) this equation becomes

$$
\begin{gathered}
g_{\alpha \beta, i} U^{\alpha \beta}+g_{\alpha \beta} U_{, i}^{\alpha \beta}+\left(g_{\alpha \beta, \gamma i}-g_{\sigma \beta} A_{\alpha \gamma i}^{\sigma}-g_{\alpha \sigma} A_{\beta \gamma i}^{\sigma}\right) V^{\alpha \beta \gamma} \\
+g_{\alpha \beta, \gamma} V_{, i}^{\alpha \beta \gamma}+\left(g_{\mu \nu} E_{\alpha \beta \gamma \sigma i}^{\mu \nu}+g_{\mu \nu, \eta} F_{\alpha \beta \gamma \sigma i}^{\mu \nu \eta}\right) W^{\alpha \beta \gamma \sigma}+g_{\alpha \beta, \gamma \sigma} W_{, i}^{\alpha \beta \gamma \sigma}=0
\end{gathered}
$$

and may be written in the form (19.15) as

$$
g_{\alpha \beta} U_{1}^{\alpha \beta}+g_{\alpha \beta, \gamma} V_{1}^{\alpha \beta \gamma}+g_{\alpha \beta, \gamma \sigma} W_{1}^{\alpha \beta \gamma \sigma}=0 \text {. }
$$

By covariant differentiation of (19.21) and substitution for $g_{i j, p, q}$ and $g_{i j, p q, r}$ from (19.16) and (19.18) we again obtain a system of equations of the form (19.15). Continuing this process we are led to the following sequence of systems of equations. As in the case of the linear first integral we add to this sequence the conditions (19.3) and (19.4) and also the symmetry conditions on the $g$ 's for the purpose of convenient reference:

$$
\left\{\begin{array}{c}
g_{i j, p}+g_{j p, i}+g_{p i, j}=0 \\
g_{i j, p q}+g_{j p, i q}+g_{p i, j q}=2\left(g_{i \alpha} A_{j p q}^{\alpha}+g_{j \alpha} A_{p i q}^{\alpha}+g_{p \alpha} A_{i j q}^{\alpha}\right) \\
g_{i j}=g_{j i} ; \quad g_{i j, p}=g_{j i, p} ; \quad g_{i j, p q}=g_{j i, p q} ; \quad g_{i j, p q}=g_{i j, q p}
\end{array}\right.
$$

$$
\begin{aligned}
& g_{\alpha \beta} U^{\alpha \beta}+g_{\alpha \beta, \gamma} V^{\alpha \beta \gamma}+g_{\alpha \beta, \gamma \sigma} W^{\alpha \beta \gamma \sigma}=0, \\
& g_{\alpha \beta} U_{1}^{\alpha \beta}+g_{\alpha \beta, \gamma} V_{1}^{\alpha \beta \gamma}+g_{\alpha \beta, \gamma \sigma} W_{1}^{\alpha \beta \gamma \sigma}=0, \\
& g_{\alpha \beta} U_{n}^{\alpha \beta}+g_{\alpha \beta, \gamma} V_{n}^{\alpha \beta \gamma}+g_{\alpha \beta, \gamma \sigma} W_{n}^{\alpha \beta \gamma \sigma}=0,
\end{aligned}
$$


The algebraic consistency of the equations (19.22) is a necessary condition for the existence of a homogeneous quadratic first integral. As in the preceding cases there must therefore exist a number $N$ such that the first $N+1$ systems of equations of (19.22) possess a fundamental set of $s$ solutions $g_{i j}^{(\alpha)}$; $g_{i j p}^{(\alpha)} ; g_{i j p q}^{(\alpha)}(\alpha=1,2, \ldots s)$ each of which satisfies the $(N+2)$ nd system of the sequence. We shall show that this is also a sufficient condition for the existence of the quadratic integral (19.1).

We first take the case in which $N=1$, and $s=1$. The first two systems of equations (19.22) then possess a unique solution which satisfies (19.20). This solution possesses a tensor character so that we may substitute it in (19.15) and differentiate covariantly, obtaining

$$
g_{\alpha \beta, i} U^{\alpha \beta}+g_{\alpha \beta} U_{, i}^{\alpha \beta}+g_{\alpha \beta \gamma, i} V^{\alpha \beta \gamma}+g_{\alpha \beta \gamma} V_{, i}^{\alpha \beta \gamma}
$$

$$
+g_{\alpha \beta \gamma \sigma, i} W^{\alpha \beta \gamma \sigma}+g_{\alpha \beta \gamma \sigma} W_{, i}^{\alpha \beta \gamma \sigma}=0
$$

Subtracting (19.20), into which the solution $g_{i j} ; g_{i j p} ; g_{i j p q}$ has been substituted instead of $g_{i j} ; g_{i j, p} ; g_{i j, p q}$, from (19.23),

$$
\left(g_{\alpha \beta, i}-g_{\alpha \beta i}\right) U^{\alpha \beta}+\left(g_{\alpha \beta \gamma, i}-g_{\alpha \beta \gamma i}+g_{\sigma \beta} A_{\alpha \gamma i}^{\sigma}+g_{\alpha \sigma} A_{\beta \gamma i}^{\sigma}\right) \nabla^{\alpha \beta \gamma}
$$

$$
+\left(g_{\alpha \beta \gamma \sigma, i}-g_{\mu \nu} E_{\alpha \beta \gamma \sigma i}^{\mu \nu}-g_{\mu \nu \eta} F_{\alpha \beta \gamma \sigma i}^{\mu \nu \eta}\right) W^{\alpha \beta \gamma \sigma}=0
$$

The solution appearing in (19.24) satisfies the first system of equations (19.22) in the summed indices and is consequently given by

$$
g_{i j, p}-g_{i j p}=\varphi_{p} g_{i j}
$$

$$
g_{i j p, q}-g_{i j p q}+g_{\alpha j} A_{i p q}^{\alpha}+g_{i \alpha} A_{j p q}^{\alpha}=\varphi_{q} g_{i j p},
$$

$$
g_{i j p q, r}-g_{\alpha \beta} E_{i j p q r}^{\alpha \beta}-g_{\alpha \beta \gamma} F_{i j p q r}^{\alpha \beta \gamma}=\varphi_{r} g_{i j p q} .
$$


The covariant vector $\varphi_{i}$ is the gradient of a scalar function, for if we differentiate (19.25) covariantly,

$$
g_{i j, p, q}-g_{i j p, q}=\varphi_{p, q} g_{i j}+\varphi_{p} g_{i j, q} .
$$

Hence,

(19.28) $\left(\varphi_{p, q}-\varphi_{q, p}\right) g_{i j}=g_{i j, p, q}-g_{i j, q, p}+g_{i j g, p}-g_{i j p, q}+\varphi_{q} g_{i j, p}-\varphi_{p} g_{i j, q}$.

If we substitute the values given by (12.4), (19.25), and (19.26) for the covariant derivatives in (19.28) we find that the right member vanishes identically. In the left member of (19.28) $g_{i j}$ can not be equal to zero, for if this were so we see from (19.25) and (19.26) that $g_{i j p}$ and $g_{i j p q}$ would also vanish, which is contrary to the assumption that our equations are algebraically consistent. Hence

or

$$
\varphi_{p, q}-\varphi_{q, p}=0
$$

$$
\varphi_{p}=\frac{\partial \varphi}{\partial x^{p}}
$$

A homogeneous quadratic first integral (19.1) will exist if a function $\psi$ can be chosen so that

$$
\left(\psi g_{i j}\right)_{p}=\psi g_{i j p}
$$

where the left member denotes the covariant derivative of $\psi g_{i j}$. If we expand (19.30) we find that the condition takes the form

$$
\frac{\partial \log \psi}{\partial x^{p}}+\varphi_{p}=0
$$

Therefore (19.30) is satisfied if we put

$$
\psi=e^{-\varphi}
$$

Hence, a sufficient condition for the existence of a homogeneous quadratic first integral (19.1) is that the first two systems of equations (19.22) possess a unique solution which satisfies the third system. 
We return now to the general case and assume that there exists a number $N$ such that the first $(N+1)$ systems of equations (19.22) admit a fundamental set of $s$ solutions $g_{i j}^{(\alpha)} ; g_{i j p}^{(\alpha)} ; g_{i j p q}^{(\alpha)}(\alpha=1,2, \ldots, s)$ each of which satisfies the $(N+2)$ nd system of equations. The general solution of the first $(N+1)$ systems of equations may then be written

$$
\begin{gathered}
g_{i j}=\varphi^{(\alpha)} g_{i j}^{(\alpha)}, \\
g_{i j p}=\varphi^{(\alpha)} g_{i j p}^{(\alpha)}, \\
g_{i j p q}=\varphi^{(\alpha)} g_{i j p q}^{(\alpha)},
\end{gathered}
$$

where the right members are summed for $\alpha$ from $\alpha=1$ to $\alpha=s$. Let us substitute the particular solution $g_{i j}^{(k)} ; g_{i j p}^{(k)} ; g_{i j p q}^{(k)}$ in the equations (19.22) beginning with the second system and ending with the $(N+2)$ nd. If we then differentiate each system of equations through the $(N+1)$ st covariantly, and subtract it from the system immediately following, we shall obtain the equations

$$
\begin{aligned}
\left(g_{\alpha \beta, i}^{(k)}-g_{\alpha \beta i}^{(k)}\right) U^{\alpha \beta} & +\left(g_{\alpha \beta \gamma, i}^{(k)}-g_{\alpha \beta \gamma i}^{(k)}+g_{\sigma \beta}^{(k)} A_{\alpha \gamma i}^{\sigma}+g_{\alpha \sigma}^{(k)} A_{\beta \gamma i}^{\sigma}\right) V^{\alpha \beta \gamma} \\
& +\left(g_{\alpha \beta \gamma \sigma, i}^{(k)}-g_{\mu \nu}^{(k)} E_{\alpha \beta \gamma \sigma i}^{\mu \nu}-g_{\mu \nu \eta}^{(k)} F_{\alpha \beta \gamma \sigma i}^{\mu \nu \eta}\right) W^{\alpha \beta \gamma \sigma}=0, \\
\left(g_{\alpha \beta, i}^{(k)}-g_{\alpha \beta i}^{(k)}\right) U_{1}^{\alpha \beta} & +\left(g_{\alpha \beta \gamma, i}^{(k)}-g_{\alpha \beta \gamma i}^{(k)}+g_{\sigma \beta}^{(k)} A_{\alpha \gamma i}^{\sigma}+g_{\alpha \sigma}^{(k)} A_{\beta \gamma i}^{\sigma}\right) V_{1}^{\alpha \beta \gamma} \\
& +\left(g_{\alpha \beta \gamma \sigma, i}^{(k)}-g_{\mu \nu}^{(k)} E_{\alpha \beta \gamma \sigma i}^{\mu \nu}-g_{\mu \nu \eta}^{(k)} F_{\alpha \beta \gamma \sigma i}^{\mu \nu \eta}\right) W_{1}^{\alpha \beta \gamma \sigma}=0,
\end{aligned}
$$

$$
\begin{aligned}
\left(g_{\alpha \beta, i}^{(k)}-g_{\alpha \beta i}^{(k)}\right) U_{N-1}^{\alpha \beta} & +\left(g_{\alpha \beta \gamma, i}^{(k)}-g_{\alpha \beta \gamma i}^{(k)}+g_{\sigma \beta}^{(k)} A_{\alpha \gamma i}^{\sigma}+g_{\alpha \sigma}^{(k)} A_{\beta \alpha i}^{\sigma}\right) V_{N-1}^{\alpha \beta \gamma} \\
& +\left(g_{\alpha \beta \gamma \sigma, i}^{(k)}-g_{\mu \nu}^{(k)} E_{\alpha \beta \gamma \sigma i}^{\mu \nu}-g_{\mu \nu \eta}^{(k)} F_{\alpha \beta \gamma \sigma i}^{\mu \nu \eta}\right) E_{N-1}^{\alpha \beta \gamma \sigma}=0 .
\end{aligned}
$$


Since the solution which appears in (19.35) satisfies the first $(N+1)$ systems of equations (19.22), it may be written as a linear combination of the fundamental solutions $g_{i j}^{(k)} ; g_{i j p}^{(k)} ; g_{i j p q}^{(k)}, k=1,2, \ldots, s$. Hence

$$
g_{i j, p}^{(k)}-g_{i j p}^{(k)}=\lambda_{p}^{(k \alpha)} g_{i j}^{(\alpha)}
$$

$$
\begin{aligned}
g_{i j p, q}^{(k)}-g_{i j p q}^{(k)}+g_{\sigma j}^{(k)} A_{i p q}^{\sigma}+g_{i \sigma}^{(k)} A_{j p q}^{\sigma} & =\lambda_{q}^{(k \alpha)} g_{i j p}^{(\alpha)}, \\
g_{i j p q, r}^{(k)}-g_{\mu \nu}^{(k)} E_{i j p q r}^{\mu \nu}-g_{\mu \nu \eta}^{(k)} F_{i j p q r}^{\mu \nu \eta} & =\lambda_{r}^{(k \alpha)} g_{i j p q}^{(\alpha)},
\end{aligned}
$$

the left members of these equations being summed for $\alpha$ from $\alpha=1$ to $\alpha=s$. To find the conditions which the covariant vectors $\lambda$ must satisfy we proceed in the same way as for the linear integral and thus obtain the equations

$$
\begin{aligned}
& g_{i j}^{(\beta)}\left(\lambda_{p, q}^{(\alpha \beta)}-\lambda_{q, p}^{(\alpha \beta)}+\lambda_{p}^{(\alpha \gamma)} \lambda_{q}^{(\gamma \beta)}-\lambda_{q}^{(\alpha \gamma)} \lambda_{p}^{(\gamma \beta)}\right)=0, \\
& g_{i j p}^{(\beta)}\left(\lambda_{q, r}^{(\alpha \beta)}-\lambda_{r, q}^{(\alpha \beta)}+\lambda_{q}^{(\alpha \gamma)} \lambda_{r}^{(\gamma \beta)}-\lambda_{r}^{(\alpha \gamma)} \lambda_{q}^{(\gamma \beta)}\right)=0, \\
& \alpha_{i j p q}^{(\beta)}\left(\lambda_{r, s}^{(\alpha \beta)}-\lambda_{s, r}^{(\alpha \beta)}+\lambda_{r}^{(\alpha \gamma)} \lambda_{s}^{(\gamma \beta)}-\lambda_{s}^{(\alpha \gamma)} \lambda_{r}^{(\gamma \beta)}\right)=0,
\end{aligned}
$$

which are summed for $\beta$ from $\beta=1$ to $\beta=s$. It follows consequently that

$$
\lambda_{p, q}^{(\alpha \beta)}-\lambda_{q, p}^{(\alpha \beta)}+\lambda_{p}^{(\alpha \gamma)} \lambda_{q}^{(\gamma \beta)}-\lambda_{q}^{(\alpha \gamma)} \lambda_{p}^{(\gamma \beta)}=0
$$

for otherwise one of the fundamental solutions could be expressed linearly in terms of the others.

A homogeneous quadratic first integral (19.1) will exist provided that the arbitrary functions $\varphi$ in the general solution can be so chosen that

$$
\left(\varphi^{(\alpha)} g_{i j}^{(\alpha)}\right)_{p}=\varphi^{(\alpha)} g_{i j p}^{(\alpha)}
$$


where the term on the left denotes the covariant derivative of $\varphi^{(\alpha)} g_{i j}^{(\alpha)}$. If we expand (19.41) we find that the quadratic integral will exist if the $\varphi$ 's can be chosen so as to satisfy the equation

$$
\frac{\partial \varphi^{(\beta)}}{\partial x^{p}}+\varphi^{(\alpha)} \lambda_{p}^{(\alpha \beta)}=0
$$

The integrability condition of (19.42) is the equation (19.40) so that a set of $\varphi$ 's can be found which will satisfy (19.42).

Hence, a necessary and sufficient condition for the existence of a quadratic first integral (19.1) is that the $\Gamma$ 's be such that there exists a number $N$ such that the first $(N+1)$ systems of equations (19.22) admit a fundamental set of $s$ solutions $(s \geqq 1)$ which satisfies the $(N+2)$ nd system of equations.

PRINCETON UNIVERSITY, PRINCETON, N. J. 